\title{
Article \\ Distinct Exosomal miRNA Profiles from BALF and Lung Tissue of COPD and IPF Patients
}

\author{
Gagandeep Kaur ${ }^{1}$, Krishna Prahlad Maremanda ${ }^{1}$, Michael Campos ${ }^{2}$, Hitendra S. Chand ${ }^{3}{ }^{(}$, , Feng Li ${ }^{4}$, \\ Nikhil Hirani ${ }^{4}$, M. A. Haseeb ${ }^{5}$, Dongmei Li ${ }^{6} \mathbb{1}$ and Irfan Rahman ${ }^{1, * \mathbb{C}}$
}

1 Department of Environmental Medicine, University of Rochester Medical Center, Rochester, NY 14642, USA; Gagandeep_Kaur@URMC.Rochester.edu (G.K.); mkrishnaprahlad@gmail.com or kmaremanda@bwh.harvard.edu (K.P.M.)

2 Division of Pulmonary, Allergy, Critical Care, University of Miami School of Medicine, Miami, FL 33136, USA mcampos1@med.miami.edu

3 Department of Immunology and Nanomedicine, Florida International University, Miami, FL 33199, USA; hchand@fiu.edu

4 Centre for Inflammation Research, Queen's Medical Research Institute, University of Edinburgh, Edinburgh EH16 4TJ, UK; Feng.Li@ed.ac.uk (F.L.); N.Hirani@ed.ac.uk (N.H.)

5 Department of Cell Biology, SUNY Downstate Health Sciences University, Brooklyn, NY 11203, USA; Haseeb.Siddiqi@downstate.edu

6 Clinical and Translational Science Institute (CTSI), Public Health Sciences, University of Rochester Medical Center, Rochester, NY 14642, USA; Dongmei_li@urmc.rochester.edu

* Correspondence: irfan_rahman@urmc.rochester.edu; Tel.: +1-585-275-6911

check for

updates

Citation: Kaur, G.; Maremanda, K.P.; Campos, M.; Chand, H.S.; Li, F.;

Hirani, N.; Haseeb, M.A.; Li, D.;

Rahman, I. Distinct Exosomal miRNA Profiles from BALF and Lung Tissue of COPD and IPF Patients. Int. J. Mol. Sci. 2021, 22, 11830. https://doi.org/ $10.3390 /$ ijms222111830

Academic Editors: Consuelo Borras and Cristina Mas-Bargues

Received: 25 August 2021

Accepted: 24 October 2021

Published: 31 October 202

Publisher's Note: MDPI stays neutral with regard to jurisdictional claims in published maps and institutional affiliations.

Copyright: (c) 2021 by the authors. Licensee MDPI, Basel, Switzerland. This article is an open access article distributed under the terms and conditions of the Creative Commons Attribution (CC BY) license (https:// creativecommons.org/licenses/by/ $4.0 /)$
Abstract: Chronic obstructive pulmonary disease (COPD) and idiopathic pulmonary fibrosis (IPF) are chronic, progressive lung ailments that are characterized by distinct pathologies. Early detection biomarkers and disease mechanisms for these debilitating diseases are lacking. Extracellular vesicles (EVs), including exosomes, are small, lipid-bound vesicles attributed to carry proteins, lipids, and RNA molecules to facilitate cell-to-cell communication under normal and diseased conditions Exosomal miRNAs have been studied in relation to many diseases. However, there is little to no knowledge regarding the miRNA population of bronchoalveolar lavage fluid (BALF) or the lung-tissue-derived exosomes in COPD and IPF. Here, we determined and compared the miRNA profiles of BALF- and lung-tissue-derived exosomes of healthy non-smokers, smokers, and patients with COPD or IPF in independent cohorts. Results: Exosome characterization using NanoSight particle tracking and TEM demonstrated that the BALF-derived exosomes were $\sim 89.85 \mathrm{~nm}$ in size with a yield of $\sim 2.95 \times 10^{10}$ particles $/ \mathrm{mL}$ in concentration. Lung-derived exosomes were larger in size $(\sim 146.04 \mathrm{~nm})$ with a higher yield of $\sim 2.38 \times 10^{11}$ particles $/ \mathrm{mL}$. NGS results identified three differentially expressed miRNAs in the BALF, while there was one in the lung-derived exosomes from COPD patients as compared to healthy non-smokers. Of these, miR-122-5p was three- or five-fold downregulated among the lung-tissue-derived exosomes of COPD patients as compared to healthy non-smokers and smokers, respectively. Interestingly, there were a large number (55) of differentially expressed miRNAs in the lung-tissue-derived exosomes of IPF patients compared to non-smoking controls. Conclusions: Overall, we identified lung-specific miRNAs associated with chronic lung diseases that can serve as potential biomarkers or therapeutic targets.

Keywords: exosomes; miRNA; COPD; biomarker; BALF; lungs

\section{Introduction}

Tobacco smoking remains the most prevalent preventable cause of morbidity and mortality worldwide. Comprising of more than 5000 compounds [1], cigarette smoke is the leading risk factor for developing chronic obstructive pulmonary disease (COPD) and idiopathic pulmonary fibrosis (IPF) in humans. Despite their distinct clinical features, both COPD and IPF are defined as severe, progressive airway diseases associated with increased 
risk of cancer development [2,3]. While COPD is characterized by an irreversible and progressive airflow limitation due to emphysema and bronchitis, IPF is characterized by thickening and scarring of lung tissue with fibrotic foci, thus leading to impaired diffusion capacity [3]. The current therapies for these conditions are mainly palliative, and the chief reason of this is due to limited understanding of the pathophysiology of the respective ailment $[4,5]$.

Evidence from literature suggests the role of extracellular vesicles (EVs), specifically exosomes, in the disease severity and outcome in COPD and IPF [6-10]. Exosomes are a subtype of EVs formed by an endosomal route with a diameter of 30-150 nm [11]. They function to maintain the tissue homeostasis and intracellular stability [12]. However, they also become pathosomes due to harmful stimuli (e.g., tobacco smoke) and can participate in the progression of diseases. In this respect, EVs/exosomes can cause pathological changes, including oxidative stress, chronic inflammation, apoptosis, aging, epigenetic alterations, and multi-organ dysfunction in COPD [13-16]. Interestingly, exosomes are produced and released in large numbers in the sputum, serum, and BALF of COPD patients, which makes them a useful target to develop non-invasive diagnostics in COPD. Similarly, exosomes isolated from the biological fluids cause pro-inflammatory responses in lung cells $[13,17,18]$. Previous studies have mostly compared the serum-derived exosome populations from COPD patients and healthy individuals [19-24]. In fact, we have also compared the miRNA profiles of plasma-derived EVs from COPD patients versus healthy smokers and non-smokers [13]. However, there is little to no knowledge about the BALF or the lung-tissue-derived exosome populations in COPD or IPF.

Furthermore, numerous studies have shown that circulating miRNAs are involved in the progression, development, and severity of various diseases, including COPD and IPF $[6,9,13,17,18,25,26]$. Several circulating miRNAs are also being considered as plausible targets for biomarker development $[27,28]$. Based on this, we compared the miRNA population in the BALF and lung-tissue-derived exosomes (i.e., exosome-enriched EVs) from healthy non-smokers (NS), healthy smokers (Sm), and patients with COPD and IPF in several independent cohorts to identify potential biomarkers to determine the extent of any pulmonary damage at an early stage.

\section{Results}

\subsection{Characterization of BALF and Lung-Tissue-Derived Exosomes}

Exosomes are involved with intercellular communication, thus affecting the physiological processes in various tissues $[11,29]$. Here, we analyzed the miRNA population from the BALF and lung tissue-derived exosomes isolated from non-smokers, smokers, and patients with COPD or IPF. We first isolated the BALF and lung-tissue-derived exosomes using the methods described below. We employed immunoblotting, nanoparticle tracking analysis (NTA: NanoSight 300), and transmission electron microscopy (TEM) to characterize the isolated exosomes per the regulations of ISEV [30]. We first used NTA to determine the particle concentration, size, or distribution of exosomes in isolated samples from BALF and lung tissues. The lung-derived exosomes (avg. conc. $=2.38 \pm 2.2 \times 10^{11}$ particles $/ \mathrm{mL}$ ) had a larger size $($ mean $=146.04 \mathrm{~nm})$. On the other hand, the average size of the BALF-derived exosomes was $\sim 89.85 \mathrm{~nm}$ (avg. conc. $=2.95 \pm 2.2 \times 10^{10}$ particles $/ \mathrm{mL}$ ) (Figures $1 \mathrm{i}$ and $2 \mathrm{i}$ ). TEM analysis confirmed the morphology of isolated exosomes from BALF and lung tissue samples, as shown in Figures 1ii and 2ii. It is important to mention here that we did not observe a significant change in the exosomes isolated from the BALF and lung tissues from various sub-groups (NS, Sm, COPD, and IPF). 

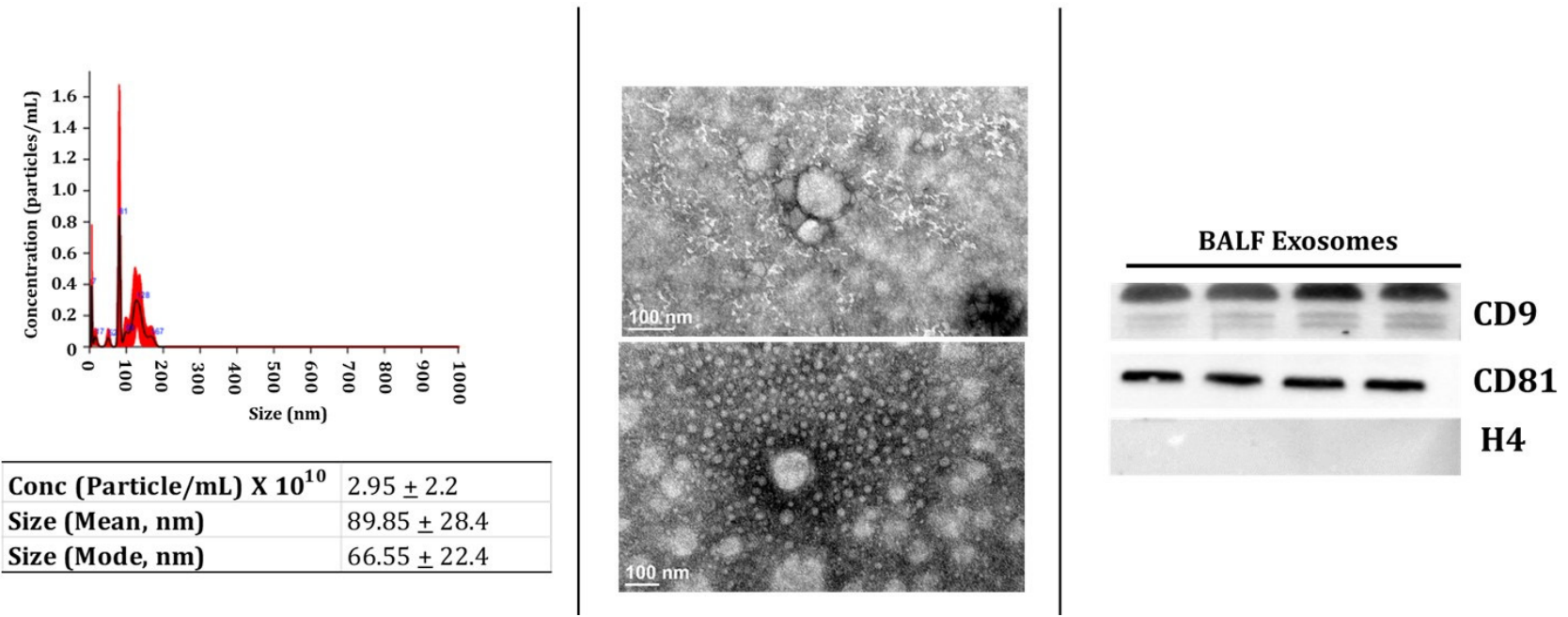

\begin{tabular}{|l|l|}
\hline Conc (Particle/mL) X 10 & $2.95 \pm 2.2$ \\
\hline Size (Mean, $\mathbf{n m )}$ & $89.85 \pm 28.4$ \\
\hline Size (Mode, $\mathbf{n m}$ ) & $66.55 \pm 22.4$ \\
\hline
\end{tabular}

Figure 1. Characterization of human BALF-derived Exosomes (i.e., exosome-enriched EVs). (i) Representative image for particle size distribution of BALF-derived exosome in one sample as estimated using NanoSight NS300. Average particle size depicted as mean, mode, and particle concentration in BALF-derived exosome samples ( $n=3-8 /$ group). (ii) Representative TEM images of BALF-derived exosomes $(n=3)$. (iii) Immunoblot analysis of positive (CD9 and CD81) and negative (H4) exosomal markers derived from human $\operatorname{BALF}(n=4)$.

(i)

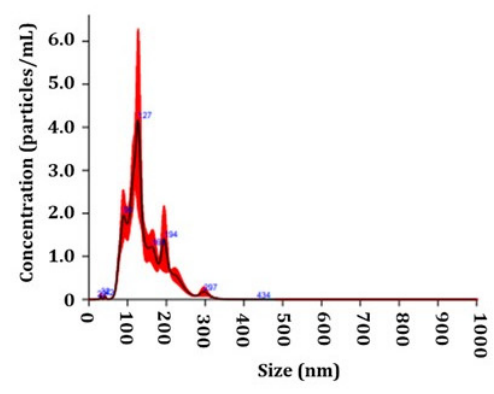

Conc (Particle/mL) X 10 $10^{11} 2.38 \pm 2.2$

Size (Mean, nm) $146.04 \pm 44.5$

Size (Mode, $\mathrm{nm}$ ) $130.56 \pm 74.0$ (ii)

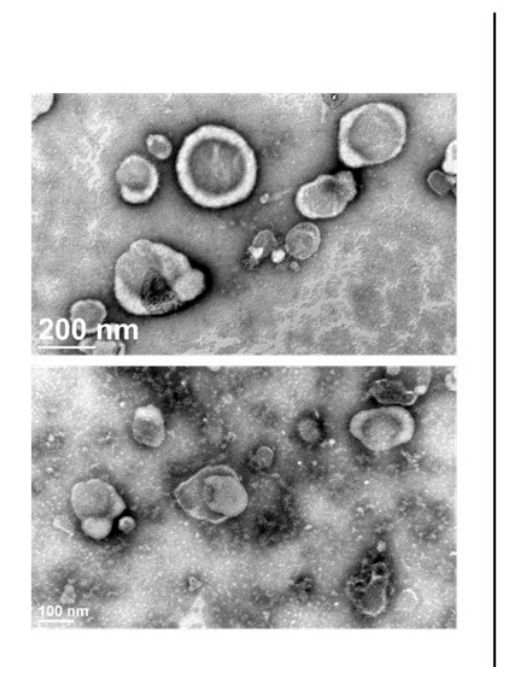

(iii)

Figure 2. Characterization of human lung-tissue-derived Exosomes (i.e., exosome-enriched EVs. (i) Representative image for particle size distribution of lung-tissue-derived exosome in one sample as estimated using NanoSight NS300. Average particle size depicted as mean, mode, and particle concentration in lung-tissue-derived exosome samples ( $n=3-5 /$ group). (ii) Representative TEM images of lung-tissue-derived exosomes $(n=6)$. (iii) Immunoblot analysis of positive (CD63 and CD81) and negative (H4) exosomal markers derived from human lung tissue $(n=4)$.

Finally, we used immunoblotting to study the presence of exosome surface markers (CD9, CD81, and CD63) in the isolated exosome fractions from the BALF and lung tissues. We found enrichment of positive surface markers for BALF exosomes, such as CD9 and CD81, in the isolated exosome fractions (Figure 1iii, full blots in Supplementary Figure S1). Similarly, we found an abundance of positive surface markers for tissue exosomes- CD63 and CD81- in the exosome fractions from the lung tissue (Figure 2iii, full blots in Supplementary Figure S2). We also probed for histone 4 (H4) for both BALF- and lung-tissue-derived exosomes as a negative marker and found no bands for this marker in 
our isolated exosomal fractions (Figures 1iii and 2iii, full blots in Supplementary Figure S3). Overall, our results confirm the successful isolation of exosomes from human BALF and lung tissue in our study groups.

\subsection{Batch Variations in the Exosome-Derived miRNA Expression Profiles amongst the Various Study Groups}

We performed principal component analyses (PCA) to visualize the batch variations within the samples. Separate analyses were run for the BALF- and lung-tissue-derived exosomes. The plot was generated by using 50 miRNAs with the highest component of variation among groups. Each sample group was clustered using a confidence ellipse, as shown in the Figure 3. The PCA plot from lung-derived exosomal miRNAs showed a distinct clustering of the IPF patient samples as compared to the other three study groups, thus suggesting a unique transcriptomic identity of these lung-derived exosomes.

(i)

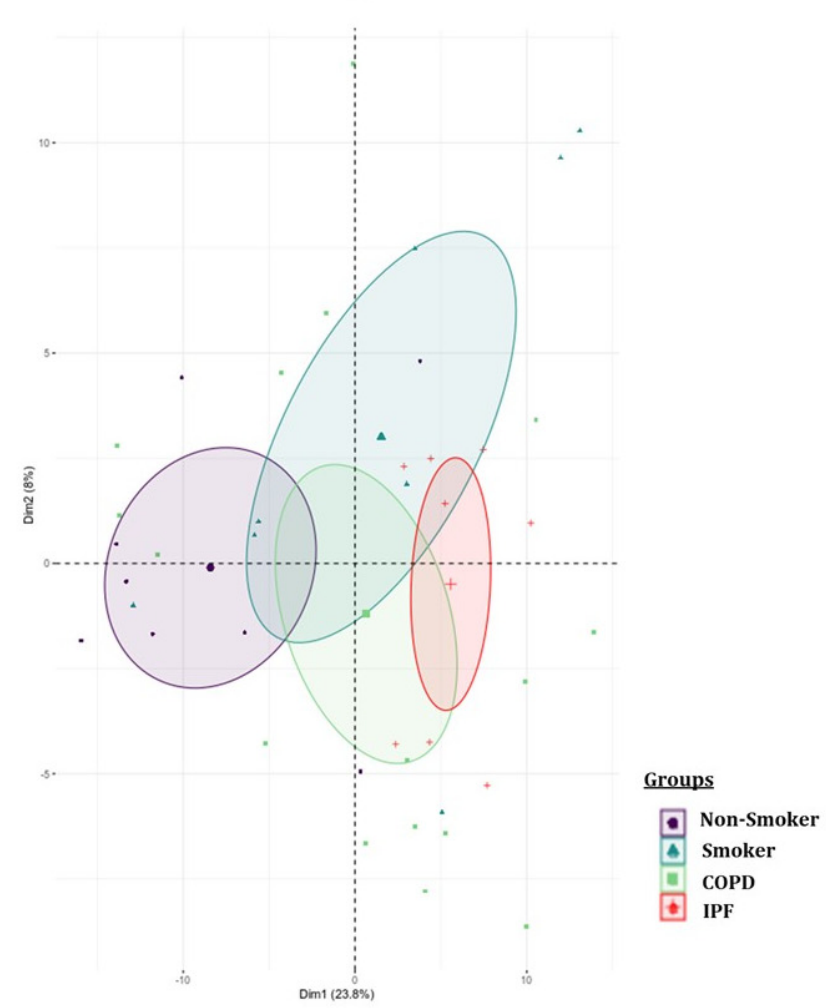

(ii)

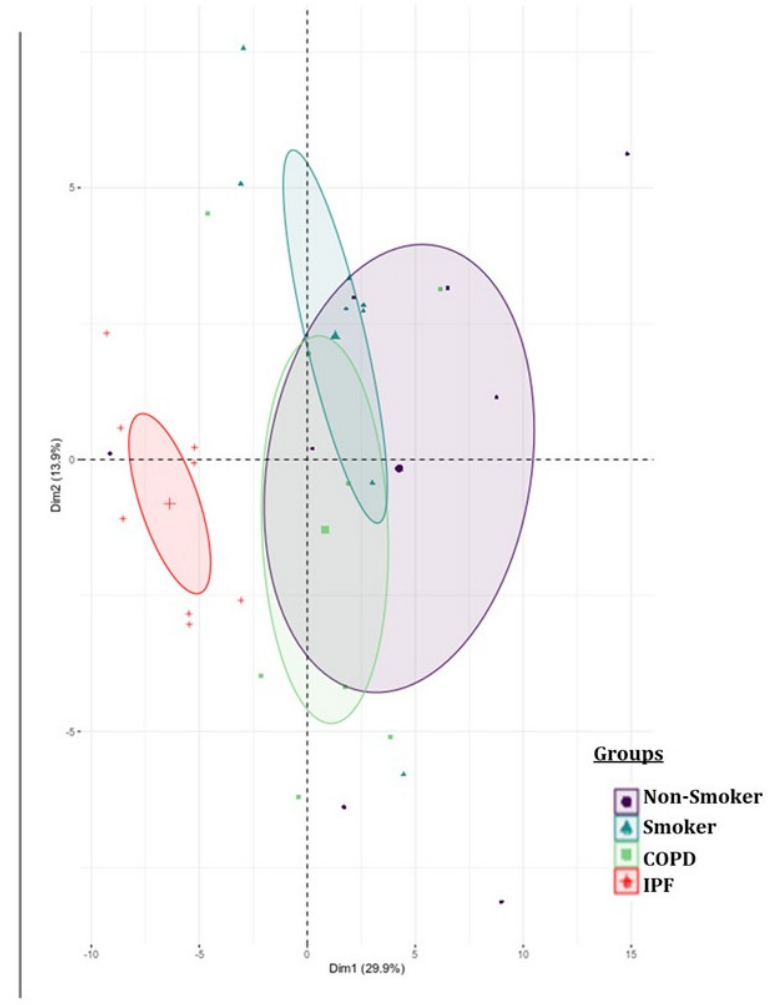

Figure 3. Principal component plot. Principal component analyses based on differential microRNA expression in individual (i) BALF- and (ii) lung-tissue-derived exosome samples from non-smokers, cigarette smokers, and COPD and IPF subjects.

\subsection{Pairwise Comparison of BALF- and Lung-Tissue-Derived Exosomal miRNA Expression Profiles}

Next, we generated volcano plots showing pairwise comparisons of the differential miRNA expression profiles between various experimental groups in BALF or lung-tissuederived exosomes (Figures 4 and 5). We plotted the - $\log _{10}$ of adjusted p-values on the $y$-axis and the $\log _{2}$ fold change between two experimental groups on the $x$-axis to generate a volcano plot. Fold changes greater than \pm 1 on the logarithmic (base2) scale of the derived volcano plots were considered significant. The miRNAs showing no significant fold change were denoted with blue, while significantly up- or downregulated miRNAs were denoted with green- and red-colored dots respectively. 
(i) NS vs Sm

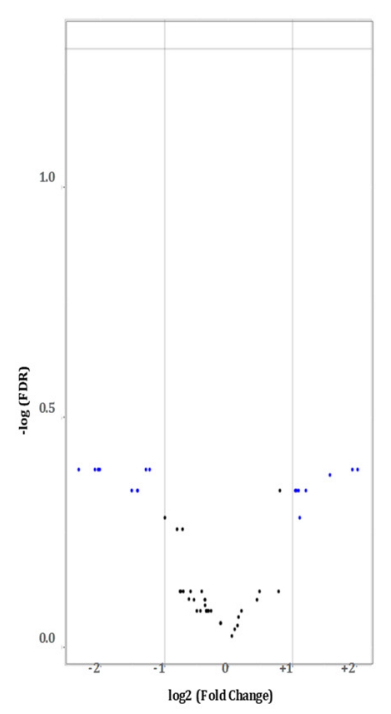

(ii) NS vs COPD

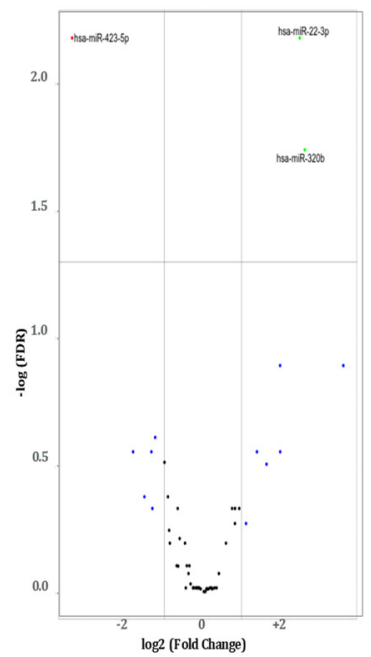

(iii) Sm vs COPD

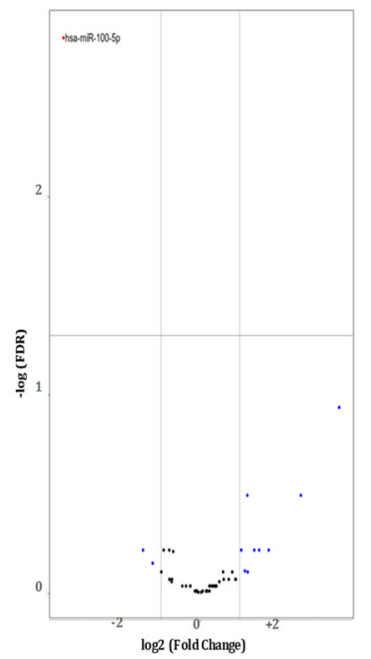

(iv) NS vs IPF

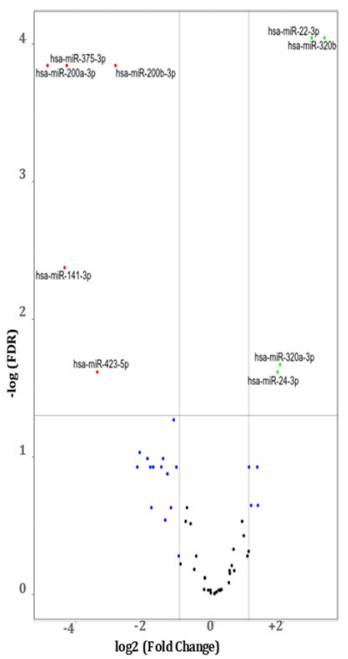

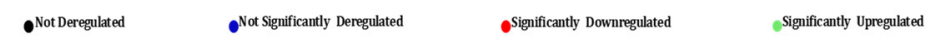

Figure 4. Volcano plots showing number and distribution of miRNAs from BALF-derived exosomes. Volcano plots showing the relation between $-\log (\mathrm{FDR})$ ( $\mathrm{y}$-axis) vs. $\log 2$ (fold change) (x-axis) in the differentially expressed miRNAs amongst BALF exosomes derived from (i) healthy non-smokers (NS) vs. healthy cigarette smokers (Sm), (ii) healthy non-smokers (NS) vs. COPD patients (COPD), (iii) healthy cigarette smokers (Sm) vs. COPD patients, and (iv) healthy non-smokers (NS) and IPF patients (IPF).

(i) NS vs Sm

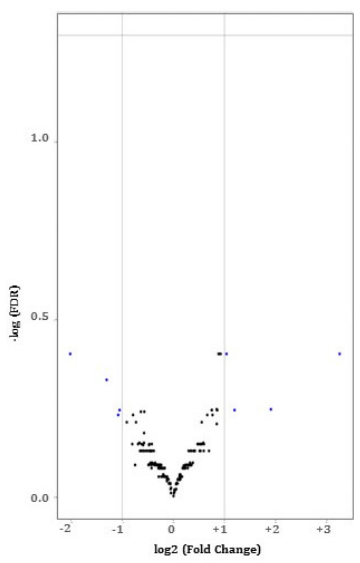

(ii) NS vs COPD

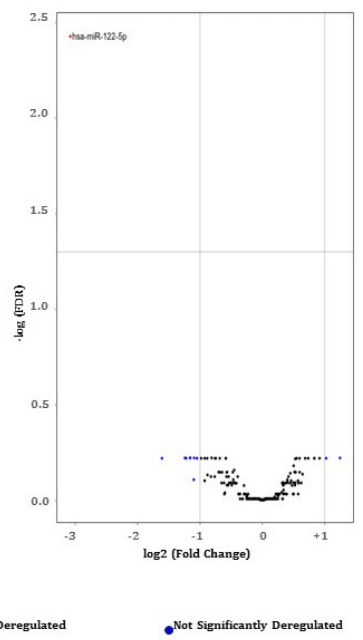

(iii) Sm vs COPD

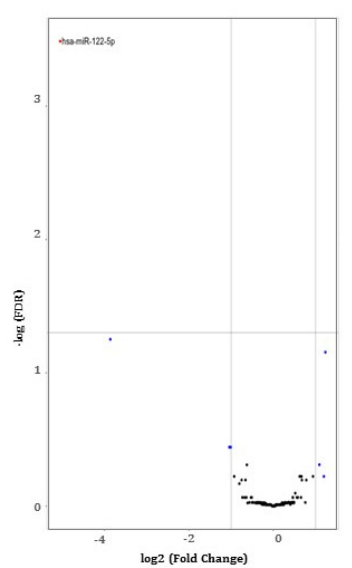

(iv) NS vs IPF

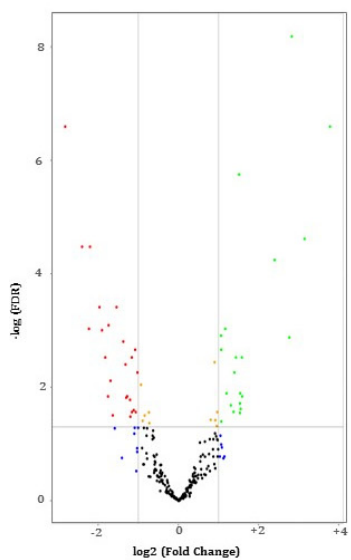

Figure 5. Volcano plots showing number and distribution of miRNAs from lung-tissue-derived exosomes. Volcano plots showing the relation between $-\log ($ FDR) (y-axis) vs. $\log 2$ (fold change) (x-axis) in the differentially expressed miRNAs amongst lung tissue derived exosomes from (i) healthy non-smokers (NS) vs. healthy cigarette smokers (Sm), (ii) healthy non-smokers (NS) vs. COPD patients (COPD), (iii) healthy cigarette smokers (Sm) vs. COPD patients, and (iv) healthy non-smokers (NS) and IPF patients (IPF). To avoid the overlap between the miRNA names, an unlabeled graph for this comparison is used. However, a detailed account of each of the differentially expressed miRNAs is provided in Figure 7.

\subsection{Hierarchical Clustering Identified Differentially Expressed miRNAs in the BALF or} Lung-Tissue-Derived Exosomes from Non-Smokers, Smokers, and Patients of COPD and IPF

We generated heat maps showing the top 50 differentially expressed miRNAs from the BALF- and lung-tissue-derived exosomes from NS vs. Sm, NS vs. COPD, NS vs. IPF, and Sm vs. COPD, as shown in Figures 6 and 7. Each miRNA is depicted in the 
individual rows of the heat map while the color scale represents the relative expression level as denoted in the scale bar alongside. Detailed information about the significantly altered miRNAs with their respective p-values and biological significance is listed in Supplementary Tables S1 and S2. In brief, the following observations were made when comparing the various experimental pairs:

(i) NS vs Sm

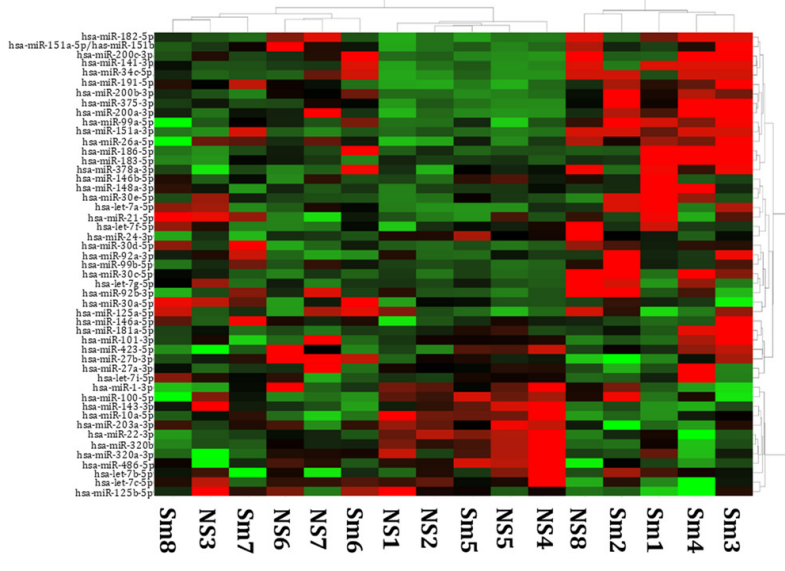

(iii) Sm vs COPD

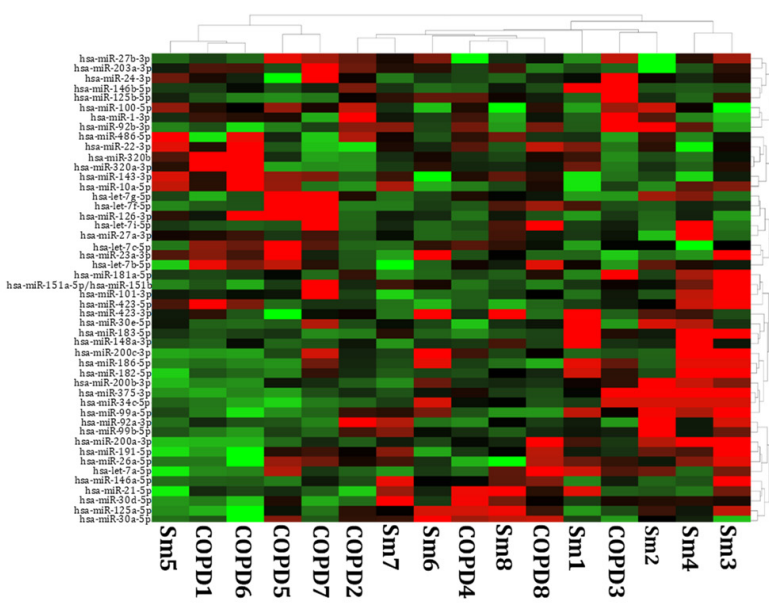

(ii) NS vs COPD
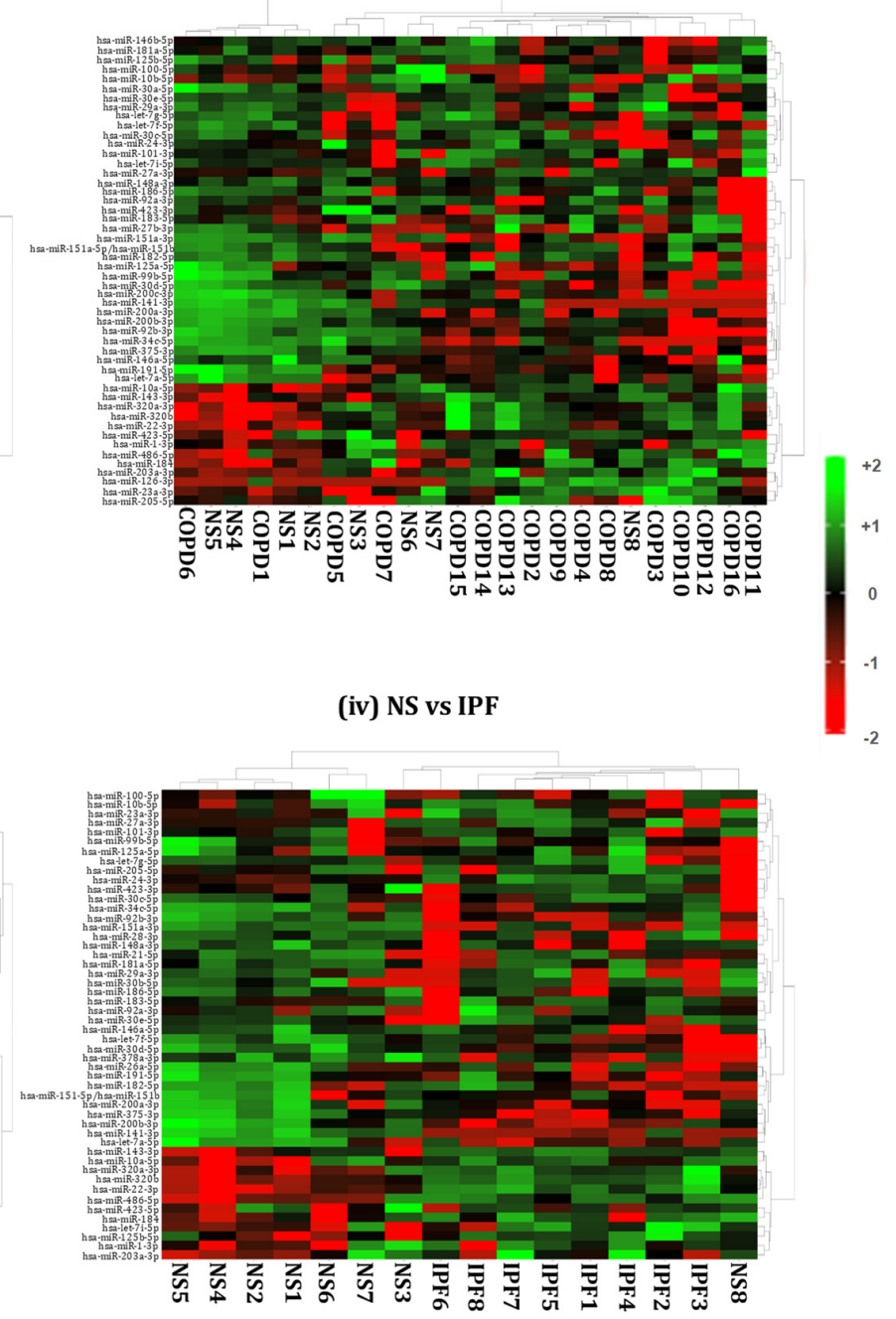

Figure 6. Hierarchical cluster analyses of differentially expressed miRNAs from BALF-derived exosomes. Heat maps showing top 50 variable miRNAs that are differentially expressed in the BALF-derived exosomes from (i) healthy nonsmokers (NS) vs. healthy smokers (Sm), (ii) healthy non-smokers (NS) vs. COPD patients (COPD), (iii) healthy smokers (Sm) vs. COPD patients (COPD), and (iv) healthy non-smokers (NS) vs. IPF patients (IPF). 
(i) NS vs Sm

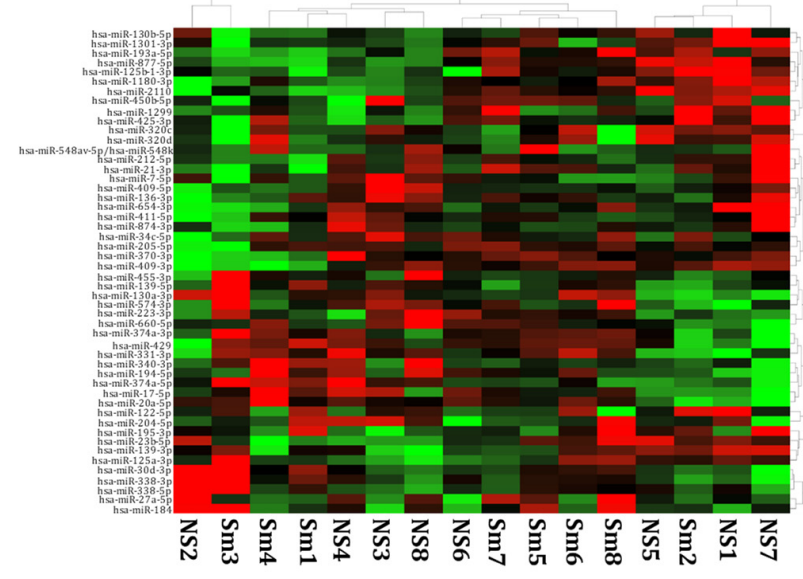

(iii) Sm vs COPD

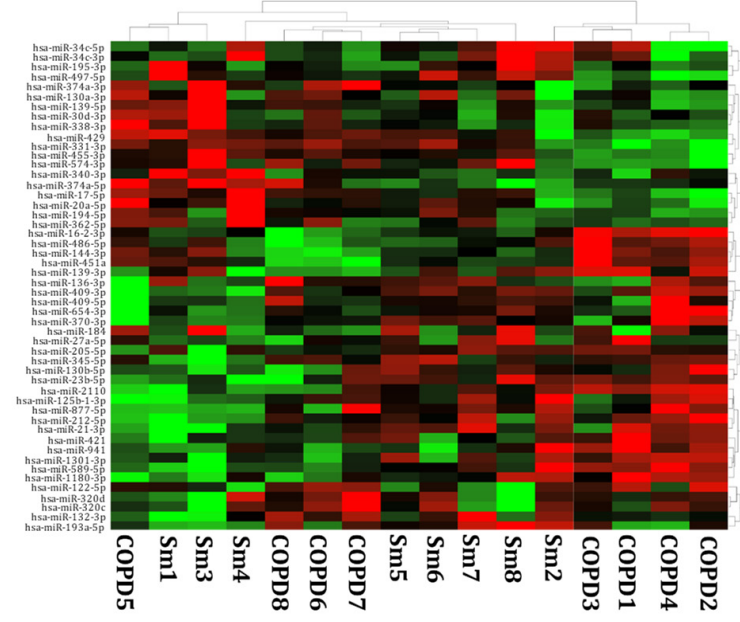

(ii) NS vs COPD

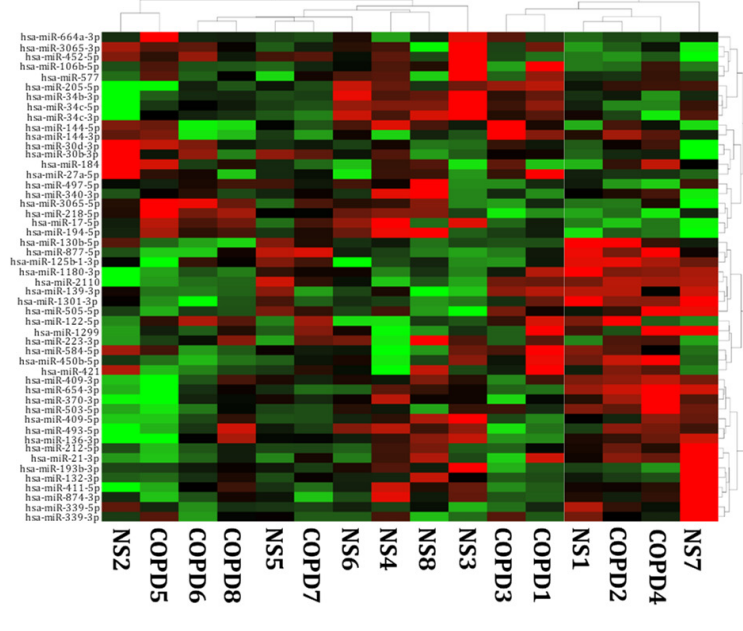

(iv) NS vs IPF

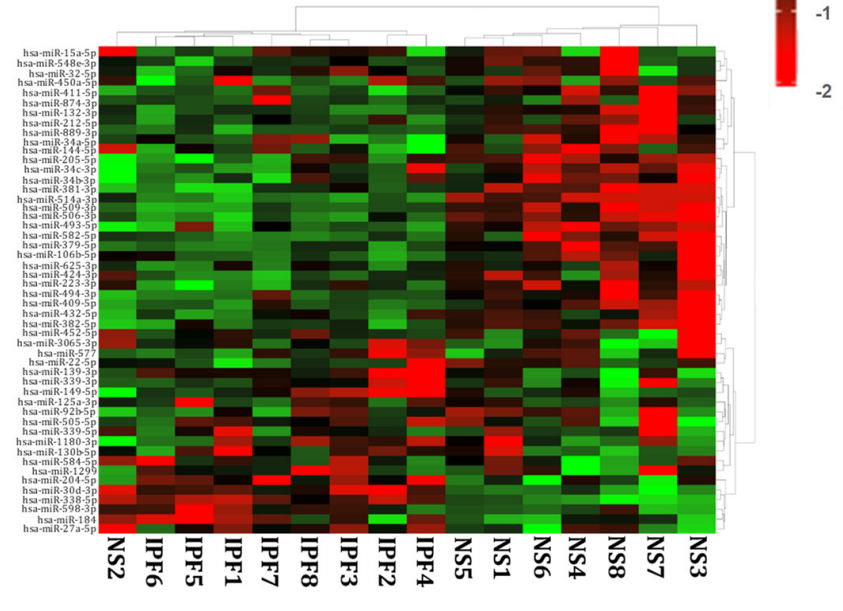

Figure 7. Hierarchical cluster analyses of differentially expressed miRNAs from lung-tissue-derived exosomes. Heat maps showing top 50 variable miRNAs that are differentially expressed in the lung-tissue-derived exosomes from (i) healthy non-smokers (NS) vs. healthy smokers (Sm), (ii) healthy non-smokers (NS) vs. COPD patients (COPD), (iii) healthy smokers (Sm) vs. COPD patients (COPD), and (iv) healthy non-smokers (NS) vs. IPF patients (IPF).

Non-smokers vs. Smokers: We did not detect any significant differentially expressed miRNAs in the BALF-derived exosomes from smokers and non-smokers. Similarly, no significant variation was observed when comparing the miRNA population from lungtissue-derived exosomes from smokers and non-smokers.

Non-smokers vs. COPD: When comparing the BALF-derived exosomal miRNAs from non-smokers and COPD patients, we found three significant differentially expressed miRNAs. Of these, two (miR-320b and miR-22-3p) were significantly upregulated, while one (miR-423-5p) was significantly downregulated in the BALF-derived exosomes from COPD patients as compared to the non-smoking controls. In contrast, we demonstrated significant downregulation of one (miR-122-5p) exosomal miRNA in the lung tissue of COPD patients as compared to non-smokers.

Smoker vs. COPD: We observed significant downregulation of miR-100-5p in the BALF-derived exosomes from COPD patients in comparison to those from healthy smokers.

Similarly, when comparing the lung-derived exosomes from these two study groups we found a significant downregulation of miR-122-5p in the exosomes derived from the lungs of COPD patients as compared to healthy smokers. Interestingly, the same miRNA 
was found to be downregulated when comparing the miRNA population from the lungderived exosomes from COPD patients and non-smokers.

Non-smokers vs. IPF: Our results showed a distinct miRNA signature in the BALFand lung-tissue-derived exosomes from IPF patients as compared to non-smoking controls. Nine differentially expressed miRNAs were identified from the BALF-derived exosomes of IPF patients as compared to healthy non-smoking controls. Of the nine, five (miR-375$3 p$, miR-200a-3p, miR-200b-3p, miR-141-3p, and miR-423-5p) miRNAs were significantly downregulated, while four (miR-22-3p, miR-320a-3p, miR-320b, and miR-24-3p) were upregulated in the BALF of IPF patients.

Interestingly, we found 55 (26 upregulated and 29 downregulated) differentially expressed miRNAs in the lung-derived exosomes from lungs of IPF patients as compared to non-smoking controls.

COPD vs. IPF: We also compared the miRNAs in BALF- and lung-derived exosomes from COPD and IPF patients. We identified one differentially expressed miRNA (miR-375$3 p$ ) in BALF-derived exosomes from IPF vs. COPD patients. Likewise, when comparing the differential miRNAs in lung tissue exosomes from COPD and IPF patients, we found 67 significant differentially (31 upregulated; 36 downregulated) expressed miRNAs in COPD patients when compared to IPF disease phenotypes. The detailed plot of these differentially expressed miRNAs and the details of the fold change are available in Supplementary Figures S4 and S5 and Supplementary Table S3.

\subsection{GO Enrichment and KEGG Analyses of Differentially Expressed miRNAs from BALF- and Lung-Derived Exosomes in COPD and IPF Patients}

To understand the potential functions of the differentially expressed miRNAs in COPD and IPF patients, we performed GO enrichment covering three major domains: biological process, cellular compartment, and molecular function. GO term annotation of differentially altered miRNAs in BALF-derived exosomes from COPD patients as compared to healthy non-smokers and smokers resulted in enrichment of terms, including posttranslational protein modification, ubiquinone biosynthetic process, cellular component organization, membrane enclosed lumen, clathrin-coated vesicle, mitochondrial matrix, protein binding, protein heterodimerization, and transferase activity. The regulatory pathway annotation via KEGG enrichment analyses showed representation of pathways involved in terpenoid backbone biosynthesis, cAMP signaling, cellular senescence, and chemokine signaling amongst COPD patients. However, none of these pathways was significantly over-represented in our analyses. GO annotation for miRNA populations obtained from IPF patient BALF resulted in enrichment of terms, including lipid transport, mesenchymal cell development, chromatin, mitochondria, lysosome, R-SMAD binding, and ATPase activity. The KEGG analyses for this subject group showed $80 \%$ overlap with the pathways enriched amongst COPD patients. Interestingly, however, we found a significant over-representation of pathways regulating glycosaminoglycan biosynthesis $(p=0.028)$ in the BALF-derived exosomes from IPF patients.

GO annotation of differentially regulated miRNAs from lung-derived exosomes was conducted separately. We found enrichment of terms like blood vessel morphogenesis, angiogenesis, transmembrane signaling receptor activity, G-protein-coupled receptor activity, calcium mediated signaling, and calcineurin-NFAT signaling cascade in lung-derived exosomes from COPD patients as compared to healthy individuals (non-smokers and smokers). Contrarily, GO terms, including plasma-membrane-bounded cell projection organization, chemical homeostasis, G-protein-coupled receptor activity, positive regulation of phospholipase $\mathrm{C}$ activity, MHC class II protein complex signaling, GTPase activator activity, and positive regulation of non-membrane spanning protein tyrosine kinase activity, were found to be enriched when analyzing differentially expressed miRNAs from lung-derived exosomes in IPF patients. KEGG enrichment analyses showed over-representation of pathways regulating apoptosis, asthma, and the cGMP-PKG signaling pathway, amongst others, in COPD patients. However, none of these regulatory pathways were significantly represented. Contrarily, KEGG enrichment analyses of miRNA profiles from IPF patients 
identified representation of 40 pathways, of which 12 were significantly represented in the miRNA population from the lung-derived exosomes from IPF patients.

Tables 1-4 provide an account of the GO enrichment and KEGG analyses results for our comparisons of various subject groups in this study. Only selected pathways are represented in the Tables.

Table 1. GO enrichment analysis of differentially expressed miRNAs in BALF-derived exosomes.

\begin{tabular}{|c|c|c|c|c|}
\hline ID & Term & Ontology & $n$ & $p$-Value * \\
\hline \multicolumn{5}{|l|}{ NS vs. COPD } \\
\hline GO:0016043 & Cellular component organization & $\mathrm{BP}$ & 17 & 0.183535762 \\
\hline GO:0071840 & Cellular component organization or biogenesis & $\mathrm{BP}$ & 17 & 0.183535762 \\
\hline GO:0061024 & Membrane organization & $\mathrm{BP}$ & 3 & 0.149797571 \\
\hline GO:0051259 & Protein complex oligomerization & $\mathrm{BP}$ & 3 & 0.149797571 \\
\hline GO:0006720 & Isoprenoid metabolic process & $\mathrm{BP}$ & 2 & 0.101214575 \\
\hline GO:0051186 & Co-factor metabolic process & $\mathrm{BP}$ & 2 & 0.101214575 \\
\hline GO:0051188 & Co-factor biosynthetic process & $\mathrm{BP}$ & 2 & 0.101214575 \\
\hline GO:0051262 & Protein tetramerization & $\mathrm{BP}$ & 2 & 0.101214575 \\
\hline GO:0008299 & Isoprenoid biosynthetic process & $\mathrm{BP}$ & 2 & 0.101214575 \\
\hline GO:0006732 & Co-enzyme metabolic process & $\mathrm{BP}$ & 1 & 0.051282051 \\
\hline GO:0009108 & Co-enzyme biosynthetic process & $\mathrm{BP}$ & 1 & 0.051282051 \\
\hline GO:0006733 & Oxidoreduction coenzyme metabolic process & $\mathrm{BP}$ & 1 & 0.051282051 \\
\hline GO:0043687 & Post-translational protein modification & $\mathrm{BP}$ & 1 & 0.051282051 \\
\hline GO:1901661 & Quinone metabolic process & $\mathrm{BP}$ & 1 & 0.051282051 \\
\hline GO:0042181 & Ketone biosynthetic process & $\mathrm{BP}$ & 1 & 0.051282051 \\
\hline GO:0051290 & Protein heterotetramerization & $\mathrm{BP}$ & 1 & 0.051282051 \\
\hline GO:0051291 & Protein heterooligomerization & $\mathrm{BP}$ & 1 & 0.051282051 \\
\hline GO:0006743 & Ubiquinone metabolic process & $\mathrm{BP}$ & 1 & 0.051282051 \\
\hline GO:0006744 & Ubiquinone biosynthetic process & $\mathrm{BP}$ & 1 & 0.051282051 \\
\hline GO:1901663 & Quinone biosynthetic process & $\mathrm{BP}$ & 1 & 0.051282051 \\
\hline GO:0031974 & Membrane-enclosed lumen & $\mathrm{CC}$ & 11 & 0.007422402 \\
\hline GO:0070013 & Intracellular organelle lumen & $\mathrm{CC}$ & 11 & 0.007422402 \\
\hline GO:0030659 & Cytoplasmic vesicle membrane & $\mathrm{CC}$ & 5 & 0.24291498 \\
\hline GO:0044431 & Golgi apparatus part & $\mathrm{CC}$ & 5 & 0.24291498 \\
\hline GO:0044429 & Mitochondrial part & $\mathrm{CC}$ & 3 & 0.149797571 \\
\hline GO:1902494 & Catalytic complex & $\mathrm{CC}$ & 3 & 0.149797571 \\
\hline GO:1990234 & Transferase complex & $\mathrm{CC}$ & 2 & 0.101214575 \\
\hline GO:0005788 & Endoplasmic reticulum lumen & $\mathrm{CC}$ & 2 & 0.101214575 \\
\hline GO:0030133 & Transport vesicle & $\mathrm{CC}$ & 2 & 0.101214575 \\
\hline GO:0005802 & Trans-Golgi network & $\mathrm{CC}$ & 1 & 0.051282051 \\
\hline GO:0030135 & Coated vesicle & $\mathrm{CC}$ & 1 & 0.051282051 \\
\hline GO:0030136 & Clathrin-coated vesicle & $\mathrm{CC}$ & 1 & 0.051282051 \\
\hline
\end{tabular}


Table 1. Cont.

\begin{tabular}{|c|c|c|c|c|}
\hline ID & Term & Ontology & $n$ & $p$-Value * \\
\hline GO:0030662 & Coated vesicle membrane & $\mathrm{CC}$ & 1 & 0.051282051 \\
\hline GO:0030665 & Clathrin-coated vesicle membrane & $\mathrm{CC}$ & 1 & 0.051282051 \\
\hline GO:0005759 & Mitochondrial matrix & $\mathrm{CC}$ & 1 & 0.051282051 \\
\hline GO:0016765 & $\begin{array}{c}\text { Transferase activity, transferring alkyl, or aryl (other than } \\
\text { methyl) groups }\end{array}$ & MF & 2 & 0.101214575 \\
\hline GO:0046982 & Protein heterodimerization & MF & 1 & 0.051282051 \\
\hline GO:0000010 & Trans-hexaprenyltranstransferase activity & MF & 1 & 0.051282051 \\
\hline GO:0050347 & Trans-octaprenyltranstransferase activity & MF & 1 & 0.051282051 \\
\hline \multicolumn{5}{|l|}{ Sm vs. COPD } \\
\hline GO:0007623 & Circadian rhythm & $\mathrm{BP}$ & 4 & 0.114285714 \\
\hline GO:0048511 & Rhythmic process & $\mathrm{BP}$ & 4 & 0.114285714 \\
\hline GO:1901566 & Organonitrogen compound biosynthetic process & $\mathrm{BP}$ & 4 & 0.114285714 \\
\hline GO:1901135 & Carbohydrate derivative metabolic process & $\mathrm{BP}$ & 3 & 0.085714286 \\
\hline GO:1901137 & Carbohydrate derivative biosynthetic process & $\mathrm{BP}$ & 2 & 0.057142857 \\
\hline GO:0006022 & Aminoglycan metabolic process & $\mathrm{BP}$ & 1 & 0.028571429 \\
\hline GO:0006023 & Aminoglycan biosynthetic process & $\mathrm{BP}$ & 1 & 0.028571429 \\
\hline GO:0006024 & Glycosaminoglycan biosynthetic process & $\mathrm{BP}$ & 1 & 0.028571429 \\
\hline GO:0005794 & Glycosaminoglycan metabolic process & $\mathrm{BP}$ & 1 & 0.028571429 \\
\hline GO:0030203 & Bounding membrane of organelle & $\mathrm{CC}$ & 8 & 0.228571429 \\
\hline GO:0001904 & Organelle sub-compartment & $\mathrm{CC}$ & 6 & 0.171428571 \\
\hline GO:0044431 & Golgi apparatus & $\mathrm{CC}$ & 6 & 0.171428571 \\
\hline GO:0098588 & Golgi sub-compartment & $\mathrm{CC}$ & 5 & 0.142857143 \\
\hline GO:0098791 & Golgi membrane & $\mathrm{CC}$ & 4 & 0.114285714 \\
\hline GO:0016740 & Transferase activity & MF & 5 & 0.142857143 \\
\hline GO:0016782 & $\begin{array}{c}\text { Transferase activity, transferring sulfur-containing } \\
\text { groups }\end{array}$ & MF & 1 & 0.028571429 \\
\hline GO:0008146 & Sulfotransferase activity & MF & 1 & 0.028571429 \\
\hline GO:0034483 & Heparan sulfate sulfotransferase activity & MF & 1 & 0.028571429 \\
\hline GO:0033871 & $\begin{array}{c}\text { (Heparan } \\
\text { sulfate)-glucosamine-3-sulfotransferase-2-activity }\end{array}$ & MF & 1 & 0.028571429 \\
\hline \multicolumn{5}{|l|}{ NS vs. IPF } \\
\hline GO:0009636 & Response to toxic substance & $\mathrm{BP}$ & 2 & 0.142682927 \\
\hline GO:0097324 & Melanocyte migration & $\mathrm{BP}$ & 1 & 0.073170732 \\
\hline GO:0097324 & Melanosome organization & $\mathrm{BP}$ & 1 & 0.073170732 \\
\hline GO:0014031 & Mesenchymal cell development & $\mathrm{BP}$ & 1 & 0.073170732 \\
\hline GO:0034204 & Lipid transport & $\mathrm{BP}$ & 1 & 0.073170732 \\
\hline GO:0044429 & Mitochondrial part & $\mathrm{CC}$ & 3 & 0.010787992 \\
\hline GO:0005739 & Mitochondrion & $\mathrm{CC}$ & 5 & 0.034709193 \\
\hline GO:0030136 & Clathrin-coated vesicle & $\mathrm{CC}$ & 1 & 0.073170732 \\
\hline
\end{tabular}


Table 1. Cont.

\begin{tabular}{ccccc}
\hline ID & Term & Ontology & $n$ & $p$-Value \\
\hline GO:0000785 & Chromatin & CC & 1 & 0.073170732 \\
\hline GO:0005766 & Primary lysosome & CC & 1 & 0.073170732 \\
\hline GO:0000010 & Trans-hexaprenyltranstransferase activity & MF & 1 & 0.073170732 \\
\hline GO:0050347 & Trans-octaprenyltranstransferase activity & MF & 1 & 0.073170732 \\
\hline GO:0016887 & ATPase activity & MF & 1 & 0.073170732 \\
\hline GO:0070412 & R-SMAD binding & MF & 1 & 0.073170732 \\
\hline
\end{tabular}

${ }^{*} p$-value for genes that were significantly up- or downregulated.

Table 2. GO enrichment analysis of differentially expressed miRNAs in lung-tissue-derived exosomes.

\begin{tabular}{|c|c|c|c|c|}
\hline ID & Term & Ontology & $n$ & $p$-Value * \\
\hline \multicolumn{5}{|l|}{ NS vs. COPD } \\
\hline GO:0048514 & Blood vessel morphogenesis & $\mathrm{BP}$ & 12 & 0.068181818 \\
\hline GO:0050808 & Synapse organization & $\mathrm{BP}$ & 11 & 0.0625 \\
\hline GO:0051962 & Positive regulation of nervous system development & $\mathrm{BP}$ & 11 & 0.0625 \\
\hline GO:0044089 & Positive regulation of cellular component biogenesis & $\mathrm{BP}$ & 10 & 0.056818182 \\
\hline GO:0044430 & Cytoskeletal part & $\mathrm{CC}$ & 9 & 0.051136364 \\
\hline GO:0001525 & Angiogenesis & $\mathrm{BP}$ & 8 & 0.045454545 \\
\hline GO:0050803 & Regulation of synapse structure or activity & $\mathrm{BP}$ & 8 & 0.045454545 \\
\hline GO:0050807 & Regulation of synapse organization & $\mathrm{BP}$ & 8 & 0.045454545 \\
\hline GO:0015630 & Microtubule cytoskeleton & $\mathrm{CC}$ & 8 & 0.045454545 \\
\hline GO:0038023 & Signaling receptor activity & MF & 8 & 0.045454545 \\
\hline GO:0060089 & Molecular transducer activity & MF & 8 & 0.045454545 \\
\hline GO:0004888 & Transmembrane signaling receptor activity & MF & 7 & 0.039772727 \\
\hline GO:0004930 & G-protein-coupled receptor activity & MF & 7 & 0.039772727 \\
\hline GO:0019932 & Second-messenger-mediated signaling & $\mathrm{BP}$ & 6 & 0.034090909 \\
\hline GO:0007416 & Synapse assembly & $\mathrm{BP}$ & 6 & 0.034090909 \\
\hline GO:0045765 & Regulation of angiogenesis & $\mathrm{BP}$ & 5 & 0.028409091 \\
\hline GO:1901342 & Regulation of vasculature development & $\mathrm{BP}$ & 5 & 0.028409091 \\
\hline GO:0051963 & Regulation of synapse assembly & $\mathrm{BP}$ & 5 & 0.028409091 \\
\hline GO:0051965 & Positive regulation of synapse assembly & $\mathrm{BP}$ & 4 & 0.022727273 \\
\hline GO:0019722 & Calcium-mediated signaling & $\mathrm{BP}$ & 4 & 0.022727273 \\
\hline GO:0005815 & Microtubule organizing center & $\mathrm{CC}$ & 4 & 0.022727273 \\
\hline GO:0005813 & Centrosome & $\mathrm{CC}$ & 4 & 0.022727273 \\
\hline GO:0016525 & Negative regulation of angiogenesis & $\mathrm{BP}$ & 3 & 0.017045455 \\
\hline GO:1901343 & Negative regulation of vasculature development & $\mathrm{BP}$ & 3 & 0.017045455 \\
\hline GO:2000181 & Negative regulation of blood vessel morphogenesis & $\mathrm{BP}$ & 3 & 0.017045455 \\
\hline GO:0033173 & Calcineurin-NFAT signaling cascade & $\mathrm{BP}$ & 2 & 0.011363636 \\
\hline GO:0048016 & Inositol-phosphate-mediated signaling & $\mathrm{BP}$ & 2 & 0.011363636 \\
\hline
\end{tabular}


Table 2. Cont.

\begin{tabular}{|c|c|c|c|c|}
\hline ID & Term & Ontology & $n$ & $p$-Value * \\
\hline GO:0097720 & Calcineurin-mediated signaling & $\mathrm{BP}$ & 2 & 0.011363636 \\
\hline \multicolumn{5}{|l|}{ Sm vs. COPD } \\
\hline GO:0048514 & Blood vessel morphogenesis & $\mathrm{BP}$ & 11 & 0.071428571 \\
\hline GO:0044087 & Regulation of cellular component biogenesis & $\mathrm{BP}$ & 11 & 0.071428571 \\
\hline GO:0044089 & Positive regulation of cellular component biogenesis & $\mathrm{BP}$ & 10 & 0.064935065 \\
\hline GO:0051962 & Positive regulation of nervous system development & $\mathrm{BP}$ & 10 & 0.064935065 \\
\hline GO:0001525 & Angiogenesis & $\mathrm{BP}$ & 8 & 0.051948052 \\
\hline GO:0050803 & Regulation of synapse structure or activity & $\mathrm{BP}$ & 8 & 0.051948052 \\
\hline GO:0050807 & Regulation of synapse organization & $\mathrm{BP}$ & 8 & 0.051948052 \\
\hline GO:0044430 & Cytoskeletal part & $\mathrm{CC}$ & 7 & 0.045454545 \\
\hline GO:0038023 & Signaling receptor activity & MF & 7 & 0.045454545 \\
\hline GO:0060089 & Molecular transducer activity & MF & 7 & 0.045454545 \\
\hline GO:0019932 & Second-messenger-mediated signaling & $\mathrm{BP}$ & 6 & 0.038961039 \\
\hline GO:0007416 & Synapse assembly & $\mathrm{BP}$ & 6 & 0.038961039 \\
\hline GO:0015630 & Microtubule cytoskeleton & $\mathrm{CC}$ & 6 & 0.038961039 \\
\hline GO:0004888 & Transmembrane signaling receptor activity & MF & 6 & 0.038961039 \\
\hline GO:0045765 & Regulation of angiogenesis & $\mathrm{BP}$ & 5 & 0.032467532 \\
\hline GO:1901342 & Regulation of vasculature development & $\mathrm{BP}$ & 5 & 0.032467532 \\
\hline GO:0051963 & Regulation of synapse assembly & $\mathrm{BP}$ & 5 & 0.032467532 \\
\hline GO:0051965 & Positive regulation of synapse assembly & $\mathrm{BP}$ & 4 & 0.025974026 \\
\hline GO:0019722 & Calcium-mediated signaling & $\mathrm{BP}$ & 4 & 0.025974026 \\
\hline GO:0016525 & Negative regulation of angiogenesis & $\mathrm{BP}$ & 3 & 0.019480519 \\
\hline GO:1901343 & Negative regulation of vasculature development & $\mathrm{BP}$ & 3 & 0.019480519 \\
\hline GO:2000181 & Negative regulation of blood vessel morphogenesis & $\mathrm{BP}$ & 3 & 0.019480519 \\
\hline GO:0005815 & Microtubule organizing center & $\mathrm{CC}$ & 3 & 0.019480519 \\
\hline GO:0005813 & Centrosome & $\mathrm{CC}$ & 3 & 0.019480519 \\
\hline GO:0004930 & G-protein-coupled receptor activity & MF & 3 & 0.019480519 \\
\hline GO:0033173 & Calcineurin-NFAT signaling cascade & $\mathrm{BP}$ & 2 & 0.012987013 \\
\hline GO:0048016 & Inositol-phosphate-mediated signaling & $\mathrm{BP}$ & 2 & 0.012987013 \\
\hline GO:0097720 & Calcineurin-mediated signaling & $\mathrm{BP}$ & 2 & 0.012987013 \\
\hline \multicolumn{5}{|l|}{ NS vs. IPF } \\
\hline GO:0065008 & Regulation of biological quality & $\mathrm{BP}$ & 44 & 0.0327192 \\
\hline GO:0007399 & Nervous system development & $\mathrm{BP}$ & 36 & 0.006858696 \\
\hline GO:0048878 & Chemical homeostasis & $\mathrm{BP}$ & 18 & 0.003415 \\
\hline GO:0030030 & Cell projection organization & $\mathrm{BP}$ & 17 & 0.012599483 \\
\hline GO:0120036 & Plasma-membrane-bounded cell projection organization & $\mathrm{BP}$ & 17 & 0.01259943 \\
\hline
\end{tabular}


Table 2. Cont.

\begin{tabular}{cccccc}
\hline ID & Term & Ontology & $n$ & $\boldsymbol{p}_{\text {-Value }}^{*}$ \\
\hline GO:0044459 & Plasma membrane region & CC & 10 & 0.028105097 \\
\hline GO:0007416 & Synapse assembly & BP & 7 & 0.006212841 \\
\hline GO:0030424 & Axon & CC & 7 & 0.0488352 \\
\hline GO:0150034 & Distal axon & CC & 6 & 0.0305685 \\
\hline GO:0031349 & Positive regulation of defense response & BP & 5 & 0.016747 \\
\hline GO:0044306 & Neuron projection terminus & CC & 4 & 0.007341699 \\
\hline GO:0008092 & Cytoskeletal protein binding & MF & 4 & 0.0073417 \\
\hline GO:0004930 & G-protein-coupled receptor activity & MF & 4 & 0.083811139 \\
\hline GO:0051965 & Positive regulation of synapse assembly & BP & 4 & 0.007341699 \\
\hline GO:0010863 & Positive regulation of phospholipase C activity & BP & 2 & 0.01653348 \\
\hline GO:0043235 & Receptor complex & CC & 2 & 0.01653348 \\
\hline GO:0023026 & MHC class II protein complex binding & MF & 2 & 0.0165335 \\
\hline GO:0005096 & GTPase activator activity & MF & 2 & 0.0165335 \\
\hline GO:1903997 & Positive regulation of non-membrane spanning protein & BP & 2 & 0.01653348 \\
\hline
\end{tabular}

* $p$-value for genes that were significantly up- or down-regulated. $\mathrm{BP}=$ biological process; $\mathrm{CC}=$ cellular component, and MF $=$ molecular function.

Table 3. KEGG analyses of differentially expressed miRNAs in BALF-derived exosomes from COPD and IPF patients.

\begin{tabular}{cc}
\hline KEGG Pathway & Selected Pathway \\
\hline COPD $^{+}$ & \\
\hline path:hsa00900 & \\
path:hsa04920 & Terpenoid backbone biosynthesis \\
path:hsa00520 & Adipocytokine signaling pathway \\
path:hsa04371 & Amino sugar and nucleotide sugar metabolism \\
path:hsa04140 & AMPK signaling pathway \\
path:hsa04136 & Apelin signaling pathway \\
path:hsa01040 & Autophagy-animal \\
path:hsa04024 & Autophagy-other \\
path:hsa04218 & path:hsa04062 \\
path:hsa00534 * & Biosynthesis of unsaturated fatty acids \\
IPF & cAMP signaling pathway \\
path:hsa00900 & Cellular senescence \\
path:hsa04920 & Chemokine signaling pathway \\
path:hsa00520 & Glycosaminoglycan biosynthesis \\
path:hsa04371 & \\
path:hsa04140 & \\
path:hsa04136 & Terpenoid backbone biosynthesis \\
path:hsa01040 & Adipocytokine signaling pathway \\
path:hsa04062 & Amino sugar and nucleotide sugar metabolism \\
path: hsa05206 & Apelin signaling pathway \\
\hline
\end{tabular}

* Significantly enriched pathway; ${ }^{+}$COPD patients vs. healthy controls (non-smokers and smokers); ${ }^{\#}$ IPF patients vs. healthy non-smokers. 
Table 4. KEGG analyses of differentially expressed miRNAs in lung-tissue-derived exosomes from COPD and IPF patients.

\begin{tabular}{|c|c|}
\hline KEGG Pathway & Selected Pathway \\
\hline \multicolumn{2}{|l|}{$\mathrm{COPD}^{+}$} \\
\hline path:hsa04520 & Adherens junction \\
\hline path:hsa04920 & Adipocytokine signaling pathway \\
\hline path:hsa04261 & Adrenergic signaling in cardiomyocytes \\
\hline path:hsa04960 & Aldosterone-regulated sodium reabsorption \\
\hline path:hsa00520 & Amino sugar and nucleotide sugar metabolism \\
\hline path:hsa04215 & Apoptosis-multiple species \\
\hline path:hsa05310 & Asthma \\
\hline path:hsa05100 & Bacterial invasion of epithelial cells \\
\hline path:hsa01040 & Biosynthesis of unsaturated fatty acids \\
\hline path:hsa04260 & Cardiac muscle contraction \\
\hline path:hsa04022 & cGMP-PKG signaling pathway \\
\hline \multicolumn{2}{|l|}{ IPF $^{\#}$} \\
\hline path:hsa04972 * & Pancreatic secretion \\
\hline path:hsa04970 * & Salivary secretion \\
\hline path:hsa04911 * & Insulin secretion \\
\hline path:hsa05416 * & Viral myocarditis \\
\hline path:hsa05310 & Asthma \\
\hline path:hsa01040 & Biosynthesis of unsaturated fatty acids \\
\hline path:hsa04022 & cGMP-PKG signaling pathway \\
\hline path:hsa04014 * & Ras signaling pathway \\
\hline path:hsa04727 * & GABAergic synapse \\
\hline path:hsa05033 * & Nicotine addiction \\
\hline path:hsa04722 * & Neurotrophin signaling pathway \\
\hline path:hsa04010 * & MAPK signaling pathway \\
\hline path:hsa04151 & PI3K-Akt signaling pathway \\
\hline
\end{tabular}

* Significantly enriched pathway; ${ }^{+}$COPD patients vs. healthy controls (non-smokers and smokers); ${ }^{\#}$ IPF patients vs. healthy non-smokers.

\section{Discussion}

The role of exosomes in lung diseases has gained increasing attention in recent times due to their role in influencing intercellular communication [10,31]. They are $50-150 \mathrm{~nm}$ in diameter, membrane-bound vesicles that contain protein, DNA, mRNAs, microRNAs (miRNA), and small non-coding RNAs to regulate pleiotropic functions. They have been extensively studied with respect to tumor microenvironments and neoplastic cancers and are being studied to be targeted as diagnostic tools or therapy against drug resistance [32-34]. However, not much is known about their role in lung pathologies. Recent studies suggest that exosomes mediate cellular crosstalk in lung microenvironments and that cigarette-smokeinduced exosomes promote myofibroblast differentiation in primary lung fibroblasts [35,36]. In addition, activated exosomes (due to cigarette smoke or disease conditions) result in macrophage polarization and matrix destruction in mouse models $[37,38]$. These studies indicate that exosomes affect cell-to-cell signaling in tobacco-smoke-related disorders.

In this respect, inhalation of toxic agents from tobacco smoke might result in irreparable airway injury, leading to various lung diseases like COPD and IPF. While the etiology/cause of each of these diseases might be due to environmental factors, the disease pathologies are distinct [39]. Therefore, we were interested in understanding if the exosomal population and the exosome-derived miRNA signatures from the BALF and lung tissues of non-smokers, smokers, COPD patients, and IPF patients are unique and can be developed into effective biomarkers for the clinical diagnosis of respective pathologies. Per ISEV guidelines, the isolated exosomes were characterized [30]. Due to the non-availability of a standardized method and variation in the sample quality prior to isolation, we noticed some variations in the purity and quality of the isolated exosomes. However, none of these were below the permissible limits for exosome research. Additionally, further quality 
checks of the isolated miRNAs were done by Norgen Biotek at the time of sequencing to ascertain successful isolation of good quality miRNAs. We therefore regarded these as exosomes enriched EVs that entail to both the populations derived from BALF and lung tissue. It is also pertinent to mention here that we did not observe any changes in the exosome size or concentration from the BALF or lung tissue of non-smokers, smokers, COPD and IPF patients. No marked difference was found in the RNA or protein concentrations obtained from exosomes isolated from various experimental groups.

Results from next generation sequencing revealed no significant differentially expressed miRNAs in the BALF- or lung-tissue-derived exosomes from heathy smokers and non-smokers. This suggests that smoking status alone does not affect the exosomemediated signaling in healthy individuals. However, we found a distinct variation in the miRNA populations from BALF- and lung-tissue-derived exosomes from COPD patients in comparison to healthy non-smokers. We found a three-fold downregulation in the expression of miR-423-5p in the BALF-derived exosomes from COPD patients as compared to healthy non-smoking controls. Of note, miR-423-5p is known to be involved in the regulation of apoptosis and extracellular matrix degradation in human nucleus pulposus cells [40]. Contrary to our findings, Molina-Pinelo et al. (2014) identified increased expression of miR-423-5p in the BALF collected from COPD patients as compared to the control group. However, it is important to mention here that the control group included in this study comprised of a few ex-smokers [41]. Therefore, taken together, it can be concluded that miR-423-5p is crucial in COPD and must be studied further to understand its potential role in the pathophysiology of COPD.

Further, we observed a two-fold increase in the expression of miR-320b and miR-22-3p in the BALF-derived exosomes from COPD patients as compared to the non-smoking controls. Previous study by our group identified upregulation of both miR-320b and $\mathrm{miR}-22-3 \mathrm{p}$ in the peripheral blood-derived exosomes of COPD patients, thus indicating the significant role of these miRNAs in regulating the disease phenotype. miR-320b is the negative regulator of the mitochondrial mediator, TP53-regulated inhibitor of apoptosis (TRIAP1) and has been previously shown to be upregulated in the peripheral blood mononuclear cells (PBMCs) from COPD patients [42,43]. Similarly, miR-22-3p is reported to inhibit HDAC4 to promote Th17-mediated emphysema in cigarette-smoke (4 month)exposed C57Bl/ 6 mice lungs [44]. Serum levels of miR-22-3p are increased amongst COPD patients based on their history of smoking, thus revealing the crucial nature of this miRNA in the progression of the disease [45].

When comparing the miRNA expression of lung-tissue-derived exosomes from COPD patients and non-smokers, we observed a three-fold downregulation of miR-122-5p in the lungs of COPD patients as compared to healthy non-smoking controls. Importantly, we further observed a five-fold decrease in the expression of miR-122-5p when comparing the miRNA population from lung-derived exosomes from COPD patients versus healthy smokers. Our results are in accordance with previous literature [46-48]. For instance, Zhu et al. (2020) demonstrated the downregulation of miR-122-5p in the sputum and plasma of COPD patients and proved that it functions as a negative regulator of IL-17A production [46]. It is pertinent to mention here that though we did not find any commonly altered miRNAs in the exosomes from the BALF or lung tissue of COPD patients, we found links that associate miRNA-mediated modulation of IL17-signaling amongst the diseased individual. The role of IL-17 in the disease pathology of COPD is rapidly emerging and is known to play an important role in the regulation of chronic inflammation and emphysema/COPD $[49,50]$. Hence, our findings identify the upstream regulators of this pathway that could possibly alter the IL-17-mediated inflammation in patients with advancing disease.

Next, we identified significant downregulation of miR-100-5p in the BALF-derived exosomes from COPD patients as compared to healthy smokers. Functionally, miR-100 is linked to the regulation of epithelial-mesenchymal transition (EMT), apoptosis, and inflammation [51,52]. Furthermore, Akbas and colleagues have demonstrated downregulation of 
miR-100-5p in the serum of COPD patients when compared to healthy smokers, which is in accordance with our study results [53].

The differentially expressed miRNA population from BALF- and lung-tissue-derived exosomes in COPD and IPF was very distinct in our study. We found five significantly downregulated miRNAs (miR-200a-3p, miR-200b-3p, miR-141-3p, miR-375-3p, and miR423-3p) and four significantly upregulated miRNAs (miR-320a-3p, miR-320b, miR-22-3p and miR-24-3p) in the BALF-derived exosomes from IPF patients. Of these, miR-423-3p and miR-320b were found to be significantly dysregulated amongst COPD patients as well. Of note, existing reports suggest a role of miR-200 in the pathogenesis of IPF $[54,55]$. It has been shown that miR-200 promotes TGF- $\beta 1$-induced EMT in normal cells and its downregulation results in a fibrogenic phenotype in IPF [54]. To our knowledge, there is no existing literature associating miR-141-3p, miR-22-3p, and miR-24-3p with IPF. Thus, we, for the first time, identify the association of these miRNAs with the disease pathogenesis in IPF.

We found 55 differentially expressed miRNAs in the lung-derived exosomes from IPF patients when compared to non-smokers. Of these, many, including miR-514-3p, miR122-5p, miR-10b-5p, miR-139b-3p, miR-582-5p, miR-889-3p, miR-1-3p, miR-148a-3p, and miR-151b, have never been reported with IPF. Our study, for the first time, reports the correlation of the dysregulated expression of these miRNAs in the lung-derived exosomes from IPF patients. Of note, we observed a three-fold increase in the expression of miR-506$3 p$ in the lung-derived exosomes from IPF patients as compared to the healthy non-smoking controls. Previous work by Zhu et al. (2019) reported that miR-506-3p targets the p65 subunit of NF- $\mathrm{KB}$ to induce apoptosis and inflammation in an experimental mice model for IPF. This study concluded that miR-506-3p is a regulator of lung fibrosis [56]. Our results provide clinical evidence suggesting a crucial role of this miRNA in the pathophysiology of IPF in humans. Similarly, accumulating evidence supports the role of miR-21-5p in the disease progression of IPF [57-59]. Further, the expression of miR-21-5p is controlled by the levels of TGF- $\beta$ family proteins and SMADs, both of which are key regulators in the etiology of fibrosis [60]. All these significant differentially expressed miRNAs require validation by qPCR in a larger cohort of the patient population. Since comparing COPD and IPF groups was not the focus of this study, we do not discuss the differentially expressed miRNA populations from both these groups here. Nevertheless, the comparisons for the two disease groups were performed and the heat maps and the fold changes observed for the differentially expressed miRNAs are available in the Supplementary Materials (Supplementary Table S3). Further phenotyping of COPD and IPF will provide information on key miRNAs that are affected in larger cohorts in future studies.

Despite its novelty and relevance to translational implications, our study had some limitations. First, the sample size for each of the study groups was quite small $(n=8-16)$. Second, due to non-availability of age- and gender-matched individuals in our cohorts, we were unable to normalize for the gender and age-specific bias in our results. Age could be a major confounding factor in such a work as the miRNA profiles alter based on aging [61-63]. The etiology of both COPD and IPF is affected by age, to which some of the alterations could be attributed to increased cellular senescence and accelerated aging in the diseased individuals. Future work with age- and gender-matched subjects might be able to shed light on this possibility. Third, there was non-availability of nonsmokers/never-smokers and limited information regarding the spirometry, pack years, and smoking history of all the subjects included in this study, which may have affected the final interpretation of our findings.

Overall, this is a first study that compares the BALF- and lung-tissue-derived exosomal miRNAs from IPF and COPD patients with healthy subjects to suggest the unique miRNA signatures that could serve as potential biomarkers to identify the disease progression of these pulmonary conditions and could also be developed as therapeutic targets. Future studies will be designed to validate the findings from this work in larger cohorts and to 
understand the role of exosomal miRNAs in affecting the disease development, progression, and severity of chronic lung diseases.

\section{Materials and Methods}

\subsection{Ethics/Approval}

The human patients and the patients' data included in the study were procured from several agencies (described below), as human subject recruitment was not directly involved with this work. The procurement of human lung tissue and BALF samples as de-identified samples was approved by the Materials Transfer Agreement (Institutional Review Board, IRB) with exemption on October 5, 2021 via our RSRB office Study ID: STUDY00006571 is not research involving human subjects as defined by DHHS and FDA regulations, along with laboratory protocols by the Institutional Biosafety Committee (IBC) at the University of Rochester Medical Center, Rochester, NY. The project codes and dates of approval were as follows: project code, DRAI1 001; protocol, 004; date of approval and IRB/IBC approvals, 11 February 2017 and 29 September 2017.

All the procedures / protocols were carried out per the guidelines and regulations specified by the University of Rochester, Rochester, NY. Other approvals include: (a) IRB study number 20080326 at the University of Miami and (b) registered clinical trial (NCT04016181) ethically approved by the University of Edinburgh (07/S1102/20) and NHS Lothian 2007/R/RES/02 by 14 June 2007. Additional samples were obtained from baseline measurements of Feasibility of Retinoids for the Treatment of Emphysema (FORTE) trial participants, as described previously $[64,65]$.

\subsection{Study Population and Sample Collection}

We employed bronchoalveolar lavage fluid and lung tissue collected from healthy (Non-smokers and Smokers) and diseased (COPD and IPF) human subjects as samples for this study from seven independent cohorts (Table 5). A total of 40 BALF samples and 32 lung tissue samples were chosen for this study from multiple sources. The majority of the BALF samples used in this study were procured from a commercial provider-BioIVT (Westbury, NY, USA). The rest of the BALF samples were provided by our collaborators-Dr. Michael Campos from the Division of Pulmonary, Allergy, Critical Care at the University of Miami, Dr. Haseeb Siddiqi from the Department of Cell Biology at SUNY Downstate Health Sciences University, and Dr. Nikhil Hirani from the Center of Inflammation research at Edinburgh University, UK. The samples procured from our collaborators were validated for their disease categories based on their spirometry and clinical status.

Likewise, the lung samples were procured from three sources: (a) a commercially available resource for procurement of human tissue and organs - the National Disease Research Interchange (NDRI), (b) the NHLBI-funded bio-specimen repository - the Lung Tissue Research Consortium (LTRC), and (c) the Department of Medicine and Pathology at the University of Helsinki Hospital, Finland, as reported previously [66,67].

All the subjects included in the study were above 21 years of age. Care was taken to include equal numbers of males and females in each subject group. A detailed characteristic of the BALF and lung tissue samples used for this study is provided in Table 5. 
Table 5. Clinical characteristics of study subjects.

\begin{tabular}{|c|c|c|c|c|c|}
\hline Characteristics & Non-Smokers & Smokers & COPD/Emphysema & IPF & $p$-Value * \\
\hline \multicolumn{6}{|l|}{$B A L F$} \\
\hline $\mathrm{N}$ & 8 & 8 & 16 & 8 & \\
\hline Age (years), mean (SD) & $49.6(17.3)$ & $57.4(8.9)$ & $65.9(13.3)$ & $76.5(11.4)$ & 0.0029 \\
\hline Gender & & & & & 0.2952 \\
\hline Male n (\%) & $3(37.5)$ & $2(25)$ & $7(38.9)$ & $6(75)$ & \\
\hline Not specified & 0 & 0 & 2 & 0 & \\
\hline Smoking status & & & & & 0.9719 \\
\hline Current smoker & 0 & 6 & 2 & 0 & \\
\hline Ex-smoker & 0 & 2 & 6 & 4 & \\
\hline $\mathrm{N} / \mathrm{A}$ & 0 & 0 & 7 & 0 & \\
\hline \multicolumn{6}{|l|}{ Lung Tissue } \\
\hline $\mathrm{N}$ & 8 & 8 & 8 & 8 & \\
\hline Age (years), mean (SD) & $48.3(16.3)$ & $53.8(15.4)$ & $59.1(9.9)$ & $68.9(9.6)$ & 0.0688 \\
\hline Gender & & & & & 0.981 \\
\hline Male n (\%) & $4(50)$ & $6(75)$ & $3(37.5)$ & $5(62.5)$ & \\
\hline $\mathrm{N} / \mathrm{A}$ & 2 & 1 & 0 & 0 & \\
\hline Smoking status & & & & & $>0.9999$ \\
\hline Current smoker & 0 & 7 & 2 & 0 & \\
\hline Ex-smoker & 3 & 1 & 4 & 6 & \\
\hline $\mathrm{N} / \mathrm{A}$ & 0 & 0 & 1 & 0 & \\
\hline
\end{tabular}

\subsection{BALF Exosome Isolation}

We employed a commercially available Plasma/Serum Exosome Purification and RNA Isolation Midi Kit from Norgen Biotek (Cat\# 58500; Norgen Bioteck Corporation, Thorold, ON, Canada) to isolate exosomes from human BALF samples. BALF exosomes were isolated as per the manufacturer's protocol. In brief, a $1 \mathrm{~mL}$ BALF sample was mixed with nuclease-free water, ExoC buffer and Slurry E and incubated for 5 min at room temperature. Next, the solution was centrifuged at $2000 \mathrm{rpm}$ for $5 \mathrm{~min}$ at room temperature and the supernatant was discarded. The slurry pellet was then resuspended in ExoR buffer and incubated for $10 \mathrm{~min}$ at room temperature. Thereafter, the suspension was centrifuged at $8000 \mathrm{rpm}$ for $2 \mathrm{~min}$ at room temperature and transferred to a Mini Filter Spin column to elute the exosomal fraction. The eluted exosomes were then stored at $-80{ }^{\circ} \mathrm{C}$ until further use.

\subsection{Lung Tissue Exosome Isolation}

The tissue exosomes were isolated using the protocol described by Dooner et al. (2018) [68] with some modifications. In brief, 30-40 mg of lung tissue was chopped and lysed using $1 \times$ Liberase solution containing $0.01 \%$ DNase. The tube containing tissue lysate was left on an orbital shaker at $37^{\circ} \mathrm{C}$ for $1 \mathrm{~h}$ to allow complete digestion of lung tissue. After $1 \mathrm{~h}$ of incubation, the tissue lysate was collected. The eluate was then centrifuged at $300 \mathrm{~g}$ for $10 \mathrm{~min}$ at $4{ }^{\circ} \mathrm{C}$ to remove cell debris. Next, the supernatant was transferred to a fresh tube and centrifuged at $2000 \times g$ for $10 \mathrm{~min}$ at $4{ }^{\circ} \mathrm{C}$. Again, the supernatant was transferred to a fresh tube and centrifuged at $10,000 \times g$ for $30 \mathrm{~min}$ at $4{ }^{\circ} \mathrm{C}$ to remove larger vesicles. Afterward, the supernatant was transferred to ultracentrifuge tubes and the exosomes were pelleted at $110,000 \times g$ for $70 \mathrm{~min}$ at $4{ }^{\circ} \mathrm{C}$ using an Optima Max-XP ultracentrifuge (Beckman 
Coulter, Brea, CA, USA). At this stage, the supernatant was discarded and the pellet was re-suspended in $1 \times$ PBS prior to filtering through a $0.22 \mu \mathrm{M}$ filter. The filtrate was once again spun at $110,000 \times g$ for $70 \mathrm{~min}$ at $4{ }^{\circ} \mathrm{C}$. Finally, the supernatant was discarded and the pellet was re-suspended in $1 \mathrm{~mL} 1 \times$ PBS. This contained freshly isolated tissue exosomes that were stored at $-80^{\circ} \mathrm{C}$ for future analysis.

\subsection{Exosome Characterization}

We employed a Hitachi 7650 analytical transmission electron microscope to visualize the isolated exosomes and nanoparticle tracking analysis (NanoSight NS300) to analyze particle size and concentration, as described earlier [14,69].

We also used immunoblotting to identify exosomal markers from the isolated fraction to characterize the BALF- and lung-tissue-derived exosomes. In brief, $20 \mu \mathrm{g}$ of exosomal lysate was resolved on a 10\% sodium dodecyl sulfate (SDS)-polyacrylamide gel and electroblotted onto nitrocellulose membranes. Membranes were blocked using a 5\% blocking buffer for $1 \mathrm{~h}$ and thereafter probed overnight with antibodies for exosomal surface markers. The antibodies included Histone 4(Cat\# 2592) (Cell Signaling Technologies, Danvers, MA, USA), CD9 (Cat\# ab92726), CD63 (Cat\# ab134045) (Abcam, Waltham, MA, USA), and CD81 (Cat\# EXOAB-CD81A-1) (SBI Biosciences, Palo Alto, CA, USA). In the following days, the blots were washed and probed with appropriate secondary antibodies. Chemiluminescence was detected using the Bio-Rad ChemiDoc MP Imaging System using the SuperSignal West Femto Maximum Sensitivity Substrate (Cat\# 34096, Thermo Scientific, Waltham, MA, USA).

\subsection{Exosomal RNA Extraction}

Total RNA from BALF exosomes was isolated using an Exosomal RNA Isolation Kit (Cat\# 58000, Norgen Bioteck Corporation, Thorold, ON, Canada) as per the manufacturer's protocol. The detailed procedure has been published earlier by us [13].

Alternately, we used an miRNeasy Mini Kit (Cat\# 217004, Qiagen, Germantown, MD, USA) to isolate RNA from lung exosomes as per the manufacturer's protocol. Briefly, $700 \mu \mathrm{L}$ of QIAzol lysis buffer was mixed with $250 \mu \mathrm{L}$ of exosomal fraction and the mix was homogenized using a QIAshredder. The homogenate was then mixed with $140 \mu \mathrm{L}$ of chloroform to allow phase separation and the aqueous phase was transferred to a fresh tube. Afterward, the RNA was precipitated using 100\% ethanol and washed using RWT and RPE buffers provided with the kit. Finally, the RNA was eluted using RNase-free water and stored at $-80{ }^{\circ} \mathrm{C}$ until further use. The RNA quality and quantity were checked using an Agilent 2100 Bioanalyzer.

\subsection{Library Preparation}

The isolated RNA samples were shipped to Norgen Biotek in Canada for library preparation, sequencing, and data analyses. The library preparation was performed using the standard library preparation workflow of Norgen Biotek, including $3^{\prime}$ and $5^{\prime}$ adapter ligation, followed by reverse transcription, indexing PCR, and size selection using $6 \%$ Novex TBE Gel. In brief, a Norgen Biotek Small RNA Library Prep Kit (Cat\# 63600, Norgen Bioteck Corporation, Thorold, ON, Canada) was employed for library preparation making sure to use the same lot between each batch of samples.

Samples were quantified using both PicoGreen and an Agilent 2100 Bioanalyzer. Six $\mu \mathrm{L}$ of high-quality total RNA was mixed with $3^{\prime}$ adaptor and T4 RNA ligase 2 to set up a reaction for $3^{\prime}$ adaptor ligation per the manufacturer's protocol. This was followed by the removal of excess $3^{\prime}$ adaptor and then 10-12 $\mu \mathrm{L}$ of final eluate was mixed with $5^{\prime}$ adaptor to set up a reaction for $5^{\prime}$ adaptor ligation. Next, the reaction for cDNA synthesis was set using the obtained ligated product as input, per the manufacturer's directions, and incubated at $50{ }^{\circ} \mathrm{C}$ for $1 \mathrm{~h}$ in a thermocycler. This was followed by PCR amplification and indexing, as advised, and cleanup of final indexed PCR product using an NGS Reaction Cleanup Kit. After cleaning, the samples were run on 6\% Novex TBE Gel for 50 min at 
$140 \mathrm{~V}$. The adaptor dimer not containing any library was excised, and the sample was eluted from the gel and checked for quality as per the manual's instructions. At this stage, the library quality check was performed to estimate library size and concentration using the bioanalyzer. Samples were then pooled in equimolar ratios and further size-selected using a 3\% agarose gel cassette on the Pippin prep (Part \# SAG-CDP3010). The pool was quantified by the bioanalyzer before starting the next-generation sequencing (NGS) run.

\subsection{Next-Generation Sequencing and Data Analysis}

We employed a NextSeq 500/550 High Output Kit v2 for 75 cycles (Cat\# 20024906, Illumina, San Diego, CA, USA) to perform NGS on our pooled library. Per the manufacturer's directions, the pooled library was denatured and diluted to the required concentration of $20 \mathrm{pM}$ for optimal cluster generation. The library was then applied onto the suitable flow cell and sequenced using the Illumina NextSeq 500 sequencing platform.

The raw sequence reads were analyzed by the team of bioinformaticians at Norgen Biotek using their advanced analysis pipeline for the processing of raw counts and alignment to the endogenous genome and annotated transcriptome.

\subsection{Gene Ontology and KEGG Analyses}

The gene ontology or GO enrichment analysis [70] was performed through the examination of significant GO terms associated with the differentially expressed miRNAs for each comparison group. The analysis was performed by iteratively testing the enrichment of each annotated GO term correlated with the set of pre-selected differentially expressed genes (in our case, miRNAs) in a linear fashion. Individual enriched annotated GO terms were analyzed using a Fisher's exact test for both upregulated and downregulated genes in which GO terms with an FDR adjusted p-value threshold of 0.05 were reported as significantly relevant. The FDR is the false discovery rate generated using the Benjamini-Hochberg method, which adjusts the p-value based on the FDR. The analysis was performed separately on all three GO domains, i.e., biological process, molecular function, and cellular component.

The KEGG enrichment analysis [71] was also performed to identify the differentially expressed genes within an associated pathway for various biological processes. The analysis was performed by testing the enrichment of each biological pathway with the associated gene (or miRNA) found within the set of pre-selected differentially expressed genes. Individually enriched pathways were then contrasted and compared between the two test groups using a Fisher's exact test for both upregulated and downregulated genes within the pre-selected set of differentially expressed genes. Biological pathways with an adjusted $p$-value below 0.05 were reported.

\subsection{Statistical Analysis}

The miRNA data from various batches were normalized using the trimmed mean of M-values (TMM) normalization method [72]. The TMM normalized read counts were used for differential expression analysis. The principal component analysis (PCA) was plotted using the ggfortify function in the R software (version: 3.5.1) to produce a sample clustering plot based on miRNAs with the highest variation across all samples. The coefficient of variation $(\% \mathrm{CV})$ was calculated based on the $\log _{2}$ of TMM normalized data and then the 50 miRNAs with the highest \%CV were selected and used to generate the PCA plot. The highest two components of variation were plotted on the $\mathrm{x}$-axis (the first principal component, PCA1) and the $y$-axis (the second principal component, PCA2). Confidence ellipses and average center points were calculated and added for each sample group to further organize the biological groupings.

The EdgeR statistical software package was used for DE analysis, as described previously [73,74]. The Benjamini-Hochberg procedure [75] was then used for adjusting the false discovery rate. This allowed us to identify the significant DE when comparing two groups. The DE was considered significant if a $\log$ fold change of $\geq 1$ or $\leq-1$ at $p$-value 
and FDR of $\leq 0.05$ was reported for the miRNA target. We used the ggplot2 function in the R software (version: 3.5.1) to plot volcano plots for illustrating a large number of miRNAs and displaying the particular miRNA targets with statistically significant differential expression.

Heat maps were generated using the ComplexHeatmap function in the R software (version: 3.5.1). The coefficient of variation $(\% \mathrm{CV})$ was calculated based on $\log _{2}$ of TMM normalized data and then the 50 miRNAs with the highest $\% \mathrm{CV}$ were selected and used to generate the heat map.

A Kruskal-Wallis test was used to calculate significance for sample distribution.

Supplementary Materials: Supplementary data is available online at https://www.mdpi.com/ article/10.3390/ijms222111830/s1.

Author Contributions: G.K. and K.P.M. designed and conducted the experiments; G.K. and I.R. wrote, edited, and/or revised the manuscript; G.K. was responsible for data curation; I.R. conceptually designed the overall experiments and manuscript and acquired funding; M.C., H.S.C., F.L., N.H., and M.A.H. provided BALF and/or lung tissue and edited the manuscript; D.L. provided statistical analysis support. All authors have read and agreed to the published version of the manuscript.

Funding: The National Institutes of Health (NIH) HL137738, HL133404, ES032159, and HL135613 supported this work.

Institutional Review Board Statement: All information on relevant Institutional Review Board statement and approval numbers are given below. The procurement of human lung tissue and BALF samples as de-identified samples was approved by the Materials Transfer Agreement (Institutional Review Board, IRB) and laboratory protocols by the Institutional Biosafety Committee (IBC) at the University of Rochester Medical Center, Rochester, NY. The project codes and dates of approval were as follows: project code, DRAI1 001; protocol, 004; date of approval and IRB/IBC approvals, 11 February 2017 and 29 September 2017. Other approvals include: (a) IRB study number 20080326 at the University of Miami and (b) registered clinical trial (NCT04016181) ethically approved by the University of Edinburgh (07/S1102/20) and NHS Lothian 2007/R/RES/02 by 14 June 2007. Additional samples were obtained from baseline measurements of Feasibility of Retinoids for the Treatment of Emphysema (FORTE) trial participants, as described previously.

Informed Consent Statement: Patient consent was waived as we procured the samples collected by commercial sources or by our collaborators. Necessary informed consent was obtained by our collaborators at the University of Miami, University of Edinburgh, and SUNY Downstate Medical Center. No informed consent was necessary as discarded de-identified samples were used in this study.

Data Availability Statement: All the data included in this manuscript is available online and is free to access to all readers. The NGS data and/or analyzed files during the current study are available at Gene Expression Omnibus accession number GSE180651 (https:/ / www.ncbi.nlm.nih. gov /geo / query /acc.cgi?acc=GSE180651). All authors confirm the availability of data and materials is online/free access to readers. The manuscript is available as a pre-print on the Preprint and medRxiv servers.

Acknowledgments: The authors would like to acknowledge Isaac Sundar, for his scientific inputs. We would further like to thank Sarah McNamara, research nurse at the Centre for Inflammation Research, University of Edinburgh, for providing patient care during sample collection for this study. We would like to acknowledge Robert Foronjy, associate professor at SUNY Downstate Health Sciences University for clinical support for some of the BALF samples included in the study. We would also like to acknowledge Ashokkumar Srinivasan, for his assistance in optimization of study protocol.

Conflicts of Interest: The authors have declared that no competing interests exist. 


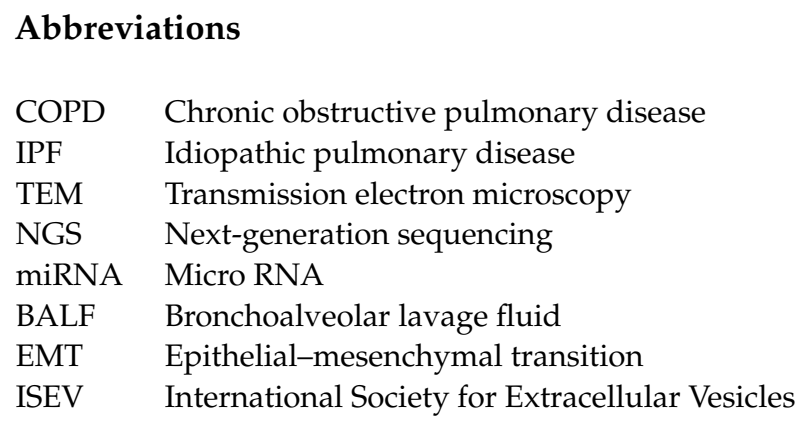

\section{References}

1. Sousa, C.; Rodrigues, M.; Carvalho, A.; Viamonte, B.; Cunha, R.; Guimarães, S.; de Moura, C.S.; Morais, A.; Pereira, J.M. Diffuse smoking-related lung diseases: Insights from a radiologic-pathologic correlation. Insights Imaging 2019, 10, 73. [CrossRef]

2. Ryu, J.; Colby, T.; Hartman, T.; Vassallo, R. Smoking-related interstitial lung diseases: A concise review. Eur. Respir. J. 2001, 17, 122-132. [CrossRef] [PubMed]

3. Chilosi, M.; Poletti, V.; Rossi, A. The pathogenesis of COPD and IPF: Distinct horns of the same devil? Respir. Res. 2012, 13, 3. [CrossRef]

4. Devine, J.F. Chronic Obstructive Pulmonary Disease: An Overview. Am. Health Drug Benefits 2008, 1, 34-42.

5. Glassberg, M.K. Overview of idiopathic pulmonary fibrosis, evidence-based guidelines, and recent developments in the treatment landscape. Am. J. Manag. Care 2019, 25, S195-S203.

6. O'Farrell, H.E.; Yang, I.A. Extracellular vesicles in chronic obstructive pulmonary disease (COPD). J. Thorac. Dis. 2019, 11, S2141-S2154. [CrossRef]

7. Wang, N.; Wang, Q.; Du, T.; Gabriel, A.N.A.; Wang, X.; Sun, L.; Li, X.; Xu, K.; Jiang, X.; Zhang, Y. The Potential Roles of Exosomes in Chronic Obstructive Pulmonary Disease. Front. Med. 2021, 7, 618506. [CrossRef]

8. Minnis, P.; Kane, R.; Anglin, R.; Walsh, S.; Worrel, J.; Khan, F.; Lumsden, R.V.; Whitty, S.; Keane, M.P. Serum exosomes from IPF patients display a fibrotic miRNA profile that correlates to clinical measures of disease severity. Eur. Respir. J. 2015, 46, PA3845. [CrossRef]

9. Njock, M.-S.; Guiot, J.; A Henket, M.; Nivelles, O.; Thiry, M.; Dequiedt, F.; Corhay, J.-L.; E Louis, R.; Struman, I. Sputum exosomes: Promising biomarkers for idiopathic pulmonary fibrosis. Thorax 2018, 74, 309-312. [CrossRef]

10. Mohan, A.; Agarwal, S.; Clauss, M.; Britt, N.S.; Dhillon, N.K. Extracellular vesicles: Novel communicators in lung diseases. Respir Res. 2020, 21, 175. [CrossRef]

11. Nederveen, J.P.; Warnier, G.; Di Carlo, A.; Nilsson, M.I.; Tarnopolsky, M.A. Extracellular Vesicles and Exosomes: Insights From Exercise Science. Front. Physiol. 2021, 11, 1757. [CrossRef]

12. Raposo, G.; Stoorvogel, W. Extracellular vesicles: Exosomes, microvesicles, and friends. J. Cell Biol. 2013, 200, 373-383. [CrossRef] [PubMed]

13. Sundar, I.K.; Li, D.; Rahman, I. Small RNA-sequence analysis of plasma-derived extracellular vesicle miRNAs in smokers and patients with chronic obstructive pulmonary disease as circulating biomarkers. J. Extracell. Vesicles 2019, 8, 1684816. [CrossRef] [PubMed]

14. Sundar, I.K.; Li, D.; Rahman, I. Proteomic Analysis of Plasma-Derived Extracellular Vesicles in Smokers and Patients with Chronic Obstructive Pulmonary Disease. ACS Omega 2019, 4, 10649-10661. [CrossRef]

15. Sundar, I.K.; Yao, H.; Rahman, I. Oxidative Stress and Chromatin Remodeling in Chronic Obstructive Pulmonary Disease and Smoking-Related Diseases. Antioxid. Redox Signal. 2013, 18, 1956-1971. [CrossRef] [PubMed]

16. Lerner, C.A.; Sundar, I.K.; Rahman, I. Mitochondrial redox system, dynamics, and dysfunction in lung inflammaging and COPD. Int. J. Biochem. Cell Biol. 2016, 81, 294-306. [CrossRef]

17. Salimian, J.; Mirzaei, H.; Moridikia, A.; Harchegani, A.B.; Sahebkar, A.; Salehi, H. Chronic obstructive pulmonary disease: MicroRNAs and exosomes as new diagnostic and therapeutic biomarkers. J. Res. Med. Sci. Off. J. Isfahan Univ. Med. Sci. 2018, 23, 27. [CrossRef]

18. Francis, S.M.S.; Davidson, M.R.; E Tan, M.; Wright, C.M.; Clarke, B.E.; E Duhig, E.; Bowman, R.V.; Hayward, N.; Fong, K.M.; A Yang, I. MicroRNA-34c is associated with emphysema severity and modulates SERPINE1 expression. BMC Genom. 2014, 15, 88. [CrossRef]

19. Takahashi, T.; Kobayashi, S.; Fujino, N.; Suzuki, T.; Ota, C.; He, M.; Yamada, M.; Suzuki, S.; Yanai, M.; Kurosawa, S.; et al Increased circulating endothelial microparticles in COPD patients: A potential biomarker for COPD exacerbation susceptibility. Thorax 2012, 67, 1067-1074. [CrossRef]

20. Porro, C.; Lacedonia, D.; Carpagnano, G.E.; Trotta, T.; Palladino, G.P.; Panaro, M.A.; Zoppo, L.D.; Barbaro, M.P.F. Microparticles in sputum of COPD patients: A potential biomarker of the disease? Int. J. Chronic Obstr. Pulm. Dis. 2016, 11, 527-533. [CrossRef]

21. Tan, D.B.; Armitage, J.; Teo, T.-H.; Ong, N.E.; Shin, H.; Moodley, Y.P. Elevated levels of circulating exosome in COPD patients are associated with systemic inflammation. Respir. Med. 2017, 132, 261-264. [CrossRef] [PubMed] 
22. Eltom, S.; Dale, N.; Raemdonck, K.; Stevenson, C.S.; Snelgrove, R.J.; Sacitharan, P.K.; Recchi, C.; Wavre-Shapton, S.; McAuley, D.; O'Kane, C.; et al. Respiratory Infections Cause the Release of Extracellular Vesicles: Implications in Exacerbation of Asthma/COPD. PLoS ONE 2014, 9, e101087. [CrossRef] [PubMed]

23. Gordon, C.; Gudi, K.; Krause, A.; Sackrowitz, R.; Harvey, B.-G.; Strulovici-Barel, Y.; Mezey, J.G.; Crystal, R.G. Circulating Endothelial Microparticles as a Measure of Early Lung Destruction in Cigarette Smokers. Am. J. Respir. Crit. Care Med. 2011, 184, 224-232. [CrossRef]

24. Shen, Y.; Wang, L.; Wu, Y.; Ou, Y.; Lu, H.; Yao, X. A novel diagnostic signature based on three circulating exosomal mircoRNAs for chronic obstructive pulmonary disease. Exp. Ther. Med. 2021, 22, 1-12. [CrossRef]

25. Guiot, J.; Njock, M.-S.; Henket, M.; Nivelles, O.; Louis, R.; Struman, I. Sputum exosomal microRNAs in IPF. Eur. Respir. J. 2018, 52, OA2119. [CrossRef]

26. Liu, B.; Li, R.; Zhang, J.; Meng, C.; Zhang, J.; Song, X.; Lv, C. MicroRNA-708-3p as a potential therapeutic target via the ADAM17-GATA/STAT3 axis in idiopathic pulmonary fibrosis. Exp. Mol. Med. 2018, 50, e465. [CrossRef] [PubMed]

27. Chen, Y.-W.R.; Leung, J.M.; Sin, D.D. A Systematic Review of Diagnostic Biomarkers of COPD Exacerbation. PLoS ONE 2016, 11, e0158843. [CrossRef] [PubMed]

28. Shaw, J.G.; Vaughan, A.; Dent, A.G.; O’Hare, P.; Goh, F.; Bowman, R.V.; Fong, K.; Yang, I.A. Biomarkers of progression of chronic obstructive pulmonary disease (COPD). J. Thorac. Dis. 2014, 6, 1532-1547. [CrossRef] [PubMed]

29. Kao, C.-Y.; Papoutsakis, E.T. Extracellular vesicles: Exosomes, microparticles, their parts, and their targets to enable their biomanufacturing and clinical applications. Curr. Opin. Biotechnol. 2019, 60, 89-98. [CrossRef] [PubMed]

30. Théry, C.; Witwer, K.W.; Aikawa, E.; Alcaraz, M.J.; Anderson, J.D.; Andriantsitohaina, R.; Antoniou, A.; Arab, T.; Archer, F.; Atkin-Smith, G.K.; et al. Minimal information for studies of extracellular vesicles 2018 (MISEV2018): A position statement of the International Society for Extracellular Vesicles and update of the MISEV2014 guidelines. J. Extracell. Vesicles 2018, 7, 1535750. [CrossRef]

31. Holtzman, J.; Lee, H. Emerging role of extracellular vesicles in the respiratory system. Exp. Mol. Med. 2020, 52, 887-895. [CrossRef]

32. Santos, P.; Almeida, F. Role of Exosomal miRNAs and the Tumor Microenvironment in Drug Resistance. Cells 2020, 9, 1450. [CrossRef] [PubMed]

33. Sun, Z.; Shi, K.; Yang, S.; Liu, J.; Zhou, Q.; Wang, G.; Song, J.; Li, Z.; Zhang, Z.; Yuan, W. Effect of exosomal miRNA on cancer biology and clinical applications. Mol. Cancer 2018, 17, 1-19. [CrossRef]

34. Ingenito, F.; Roscigno, G.; Affinito, A.; Nuzzo, S.; Scognamiglio, I.; Quintavalle, C.; Condorelli, G. The Role of Exo-miRNAs in Cancer: A Focus on Therapeutic and Diagnostic Applications. Int. J. Mol. Sci. 2019, 20, 4687. [CrossRef] [PubMed]

35. Fujita, Y.; Araya, J.; Ito, S.; Kobayashi, K.; Kosaka, N.; Yoshioka, Y.; Kadota, T.; Hara, H.; Kuwano, K.; Ochiya, T. Suppression of autophagy by extracellular vesicles promotes myofibroblast differentiation in COPD pathogenesis. J. Extracell. Vesicles 2015, 4, 28388. [CrossRef]

36. Bartel, S.; Deshane, J.; Wilkinson, T.; Gabrielsson, S. Extracellular Vesicles as Mediators of Cellular Cross Talk in the Lung Microenvironment. Front. Med. 2020, 7, 326. [CrossRef]

37. Wang, L.; Chen, Q.; Yu, Q.; Xiao, J.; Zhao, H. Cigarette smoke extract-treated airway epithelial cells-derived exosomes promote M1 macrophage polarization in chronic obstructive pulmonary disease. Int. Immunopharmacol. 2021, 96, 107700. [CrossRef]

38. Genschmer, K.R.; Russell, D.W.; Lal, C.; Szul, T.; Bratcher, P.E.; Noerager, B.D.; Roda, M.A.; Xu, X.; Rezonzew, G.; Viera, L.; et al. Activated PMN Exosomes: Pathogenic Entities Causing Matrix Destruction and Disease in the Lung. Cell 2019, 176, 113-126.e15. [CrossRef] [PubMed]

39. Fujita, Y.; Kosaka, N.; Araya, J.; Kuwano, K.; Ochiya, T. Extracellular vesicles in lung microenvironment and pathogenesis. Trends Mol. Med. 2015, 21, 533-542. [CrossRef]

40. Xu, H.; Ji, L.; Yu, C.; Chen, Q.; Ge, Q.; Lu, Y. MiR-423-5P Regulates Cells Apoptosis and Extracellular Matrix Degradation via Nucleotide-Binding, Leucine-Rich Repeat Containing X1 (NLRX1) in Interleukin 1 beta (IL-1 $\beta$ )-Induced Human Nucleus Pulposus Cells. Med. Sci. Monit. Int. Med. J. Exp. Clin. Res. 2020, 26, e922497. [CrossRef] [PubMed]

41. Molina-Pinelo, S.; Pastor, M.D.; Suarez, R.; Romero-Romero, B.; De La Pena, M.G.; Salinas, A.; Garcia-Carbonero, R.; De Miguel, M.J.; Rodriguez-Panadero, F.; Carnero, A.; et al. MicroRNA clusters: Dysregulation in lung adenocarcinoma and COPD. Eur. Respir. J. 2014, 43, 1740-1749. [CrossRef]

42. Dang, X.; Qu, X.; Wang, W.; Liao, C.; Li, Y.; Zhang, X.; Xu, D.; Baglole, C.J.; Shang, D.; Chang, Y. Bioinformatic analysis of microRNA and mRNA Regulation in peripheral blood mononuclear cells of patients with chronic obstructive pulmonary disease. Respir. Res. 2017, 18, 4. [CrossRef]

43. Li, Y.; Tang, X.; He, Q.; Yang, X.; Ren, X.; Wen, X.; Zhang, J.; Wang, Y.; Liu, N.; Ma, J. Overexpression of Mitochondria Mediator Gene TRIAP1 by miR-320b Loss Is Associated with Progression in Nasopharyngeal Carcinoma. PLoS Genet. 2016, 12, e1006183. [CrossRef] [PubMed]

44. Lu, W.; You, R.; Yuan, X.; Yang, T.; Samuel, E.; Marcano, D.C.; Sikkema, W.; Tour, J.; Rodriguez, A.; Kheradmand, F.; et al. The microRNA miR-22 inhibits the histone deacetylase HDAC4 to promote TH17 cell-dependent emphysema. Nat. Immunol. 2015, 16, 1185-1194. [CrossRef] 
45. Velasco-Torres, Y.; Ruiz, V.; Montaño, M.; Pérez-Padilla, R.; Falfán-Valencia, R.; Pérez-Ramos, J.; Pérez-Bautista, O.; Ramos, C. Participation of the miR-22-HDAC4-DLCO Axis in Patients with COPD by Tobacco and Biomass. Biomolecules $2019,9,837$. [CrossRef]

46. Zhu, K.; Zhou, S.; Xu, A.; Sun, L.; Li, M.; Jiang, H.; Zhang, B.; Zeng, D.; Fei, G.; Wang, R. Microbiota Imbalance Contributes to COPD Deterioration by Enhancing IL-17a Production via miR-122 and miR-30a. Mol. Ther.-Nucleic Acids 2020, 22, 520-529. [CrossRef]

47. Osei, E.; Florez-Sampedro, L.; Timens, W.; Postma, D.S.; Heijink, I.H.; Brandsma, C.-A. Unravelling the complexity of COPD by microRNAs: It's a small world after all. Eur. Respir. J. 2015, 46, 807-818. [CrossRef] [PubMed]

48. Qian, Y.; Mao, Z.-D.; Shi, Y.-J.; Liu, Z.-G.; Cao, Q.; Zhang, Q. Comprehensive Analysis of miRNA-mRNA-lncRNA Networks in Non-Smoking and Smoking Patients with Chronic Obstructive Pulmonary Disease. Cell. Physiol. Biochem. 2018, 50, $1140-1153$. [CrossRef] [PubMed]

49. Rich, H.E.; Alcorn, J.F. IL-17 Strikes a Chord in Chronic Obstructive Pulmonary Disease Exacerbation. Am. J. Respir. Cell Mol. Biol. 2018, 58, 669-670. [CrossRef] [PubMed]

50. Kaur, G.; Bagam, P.; Pinkston, R.; Singh, D.P.; Batra, S. Cigarette smoke-induced inflammation: NLRP10-mediated mechanisms. Toxicology 2018, 398-399, 52-67. [CrossRef]

51. Liang, Y.; Zhao, G.; Tang, L.; Zhang, J.; Li, T.; Liu, Z. MiR-100-3p and miR-877-3p regulate overproduction of IL-8 and IL-1 $\beta$ in mesangial cells activated by secretory IgA from IgA nephropathy patients. Exp. Cell Res. 2016, 347, 312-321. [CrossRef]

52. Han, W.; Ren, X.; Yang, Y.; Li, H.; Zhao, L.; Lin, Z. microRNA -100 functions as a tumor suppressor in non-small cell lung cancer via regulating epithelial-mesenchymal transition and Wnt/ $\beta$-catenin by targeting HOXA1. Thorac. Cancer 2020, 11, 1679-1688. [CrossRef] [PubMed]

53. Akbas, F.; Coskunpinar, E.; Aynac1, E.; Oltulu, Y.M.; Yildiz, P. Analysis of Serum Micro-Rnas as Potential Biomarker in Chronic Obstructive Pulmonary Disease. Exp. Lung Res. 2012, 38, 286-294. [CrossRef]

54. Yang, S.; Banerjee, S.; Freitas, A.; Sanders, Y.Y.; Ding, Q.; Matalon, S.; Thannickal, V.J.; Abraham, E.; Liu, G. Participation of miR-200 in Pulmonary Fibrosis. Am. J. Pathol. 2012, 180, 484-493. [CrossRef]

55. Moimas, S.; Salton, F.; Kosmider, B.; Ring, N.; Volpe, M.C.; Bahmed, K.; Braga, L.; Rehman, M.; Vodret, S.; Graziani, M.L.; et al. miR-200 family members reduce senescence and restore idiopathic pulmonary fibrosis type II alveolar epithelial cell transdifferentiation. ERJ Open Res. 2019, 5, 00138-02019. [CrossRef]

56. Zhu, M.; An, Y.; Zhang, X.; Wang, Z.; Duan, H. Experimental pulmonary fibrosis was suppressed by microRNA-506 through NF-kappa-mediated apoptosis and inflammation. Cell Tissue Res. 2019, 378, 255-265. [CrossRef]

57. Makiguchi, T.; Yamada, M.; Yoshioka, Y.; Sugiura, H.; Koarai, A.; Chiba, S.; Fujino, N.; Tojo, Y.; Ota, C.; Kubo, H.; et al. Serum extracellular vesicular miR-21-5p is a predictor of the prognosis in idiopathic pulmonary fibrosis. Respir. Res. 2016, 17, 110. [CrossRef] [PubMed]

58. Ma, X.; Kumar, M.; Choudhury, S.N.; Buscaglia, L.E.B.; Barker, J.R.; Kanakamedala, K.; Liu, M.-F.; Li, Y. Loss of the miR-21 allele elevates the expression of its target genes and reduces tumorigenesis. Proc. Natl. Acad. Sci. USA 2011, 108, 10144-10149. [CrossRef] [PubMed]

59. Li, Y.-F.; Jing, Y.; Hao, J.; Frankfort, N.C.; Zhou, X.; Shen, B.; Liu, X.; Wang, L.; Li, R. MicroRNA-21 in the pathogenesis of acute kidney injury. Protein Cell 2013, 4, 813-819. [CrossRef] [PubMed]

60. Davis-Dusenbery, B.; Hilyard, A.C.; Lagna, G.; Hata, A. SMAD proteins control DROSHA-mediated microRNA maturation. Nat. Cell Biol. 2008, 454, 56-61. [CrossRef]

61. Zhang, H.; Jin, K. Peripheral Circulating Exosomal miRNAs Potentially Contribute to the Regulation of Molecular Signaling Networks in Aging. Int. J. Mol. Sci. 2020, 21, 1908. [CrossRef] [PubMed]

62. D'Anca, M.; Fenoglio, C.; Serpente, M.; Arosio, B.; Cesari, M.; Scarpini, E.A.; Galimberti, D. Exosome Determinants of Physiological Aging and Age-Related Neurodegenerative Diseases. Front. Aging Neurosci. 2019, 11, 232. [CrossRef] [PubMed]

63. $\mathrm{Xu}, \mathrm{D}$.; Tahara, H. The role of exosomes and microRNAs in senescence and aging. Adv. Drug Deliv. Rev. 2013, 65, 368-375. [CrossRef]

64. Roth, M.D.; Connett, J.E.; D'Armiento, J.M.; Foronjy, R.F.; Friedman, P.J.; Goldin, J.; Louis, T.A.; Mao, J.T.; Muindi, J.R.; O'Connor, G.; et al. Feasibility of Retinoids for the Treatment of Emphysema Study. Chest 2006, 130, 1334-1345. [CrossRef] [PubMed]

65. Agudelo, C.W.; Kumley, B.K.; Area-Gomez, E.; Xu, Y.; Dabo, A.J.; Geraghty, P.; Campos, M.; Foronjy, R.; Garcia-Arcos, I. Decreased surfactant lipids correlate with lung function in chronic obstructive pulmonary disease (COPD). PLoS ONE 2020, 15, e0228279. [CrossRef]

66. Maremanda, K.P.; Sundar, I.K.; Li, D.; Rahman, I. Age-Dependent Assessment of Genes Involved in Cellular Senescence, Telomere, and Mitochondrial Pathways in Human Lung Tissue of Smokers, COPD, and IPF: Associations With SARS-CoV-2 COVID-19 ACE2-TMPRSS2-Furin-DPP4 Axis. Front. Pharmacol. 2020, 11, 11. [CrossRef] [PubMed]

67. Sundar, I.K.; Yin, Q.; Baier, B.S.; Yan, L.; Mazur, W.; Li, D.; Susiarjo, M.; Rahman, I. DNA methylation profiling in peripheral lung tissues of smokers and patients with COPD. Clin. Epigenet. 2017, 9, 1-18. [CrossRef]

68. Dooner, M.S.; Stewart, C.; Deng, Y.; Papa, E.; Pereira, M.; Del Tatto, M.; Johnson, S.; Wen, S.; Amaral, A.; Aliotta, J.; et al. Daily rhythms influence the ability of lung-derived extracellular vesicles to modulate bone marrow cell phenotype. PLoS ONE 2018, 13, e0207444. [CrossRef] 
69. Singh, K.P.; Maremanda, K.P.; Li, D.; Rahman, I. Exosomal microRNAs are novel circulating biomarkers in cigarette, waterpipe smokers, E-cigarette users and dual smokers. BMC Med. Genom. 2020, 13, 1-20. [CrossRef]

70. Ashburner, M.; Ball, C.A.; Blake, J.A.; Botstein, D.; Butler, H.; Cherry, J.M.; Davis, A.P.; Dolinski, K.; Dwight, S.S.; Eppig, J.T.; et al. Gene Ontology: Tool for the unification of biology. Nat. Genet. 2000, 25, 25-29. [CrossRef] [PubMed]

71. Kanehisa, M.; Goto, S.; Kawashima, S.; Nakaya, A. The KEGG databases at GenomeNet. Nucleic Acids Res. 2002, $30,42-46$. [CrossRef] [PubMed]

72. Robinson, M.D.; Oshlack, A. A scaling normalization method for differential expression analysis of RNA-seq data. Genome Biol. 2010, 11, R25. [CrossRef] [PubMed]

73. Robinson, M.D.; Smyth, G.K. Moderated statistical tests for assessing differences in tag abundance. Bioinformatics 2007, 23, 2881-2887. [CrossRef] [PubMed]

74. Robinson, M.D.; McCarthy, D.J.; Smyth, G.K. edgeR: A Bioconductor package for differential expression analysis of digital gene expression data. Bioinformatics 2010, 26, 139-140. [CrossRef]

75. Benjamini, Y.; Hochberg, Y. Controlling the False Discovery Rate: A Practical and Powerful Approach to Multiple Testing. J. R. Stat. Soc. Ser. B 1995, 57, 289-300. [CrossRef] 